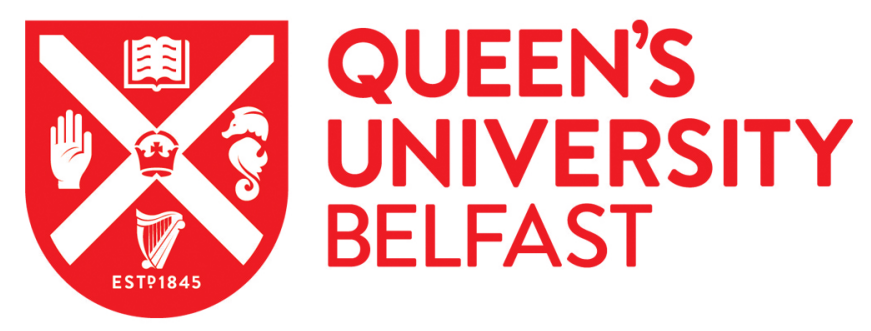

\title{
An inventory of atomic species in the atmosphere of WASP-121b using UVES high-resolution spectroscopy
}

Merritt, S. R., Gibson, N. P., Nugroho, S. K., de Mooij, E. J. W., Hooton, M. J., Lothringer, J. D., Matthews, S. M., Mikal-Evans, T., Nikolov, N., Sing, D. K., \& Watson, C. A. (2021). An inventory of atomic species in the atmosphere of WASP-121b using UVES high-resolution spectroscopy. Monthly Notices of the Royal Astronomical Society, Advance Access. https://doi.org/10.1093/mnras/stab1878

Published in:

Monthly Notices of the Royal Astronomical Society, Advance Access

Document Version:

Publisher's PDF, also known as Version of record

Queen's University Belfast - Research Portal:

Link to publication record in Queen's University Belfast Research Portal

Publisher rights

Copyright 2021 the authors.

This is an open access article published under a Creative Commons Attribution License (https://creativecommons.org/licenses/by/4.0/), which permits unrestricted use, distribution and reproduction in any medium, provided the author and source are cited.

\section{General rights}

Copyright for the publications made accessible via the Queen's University Belfast Research Portal is retained by the author(s) and / or other copyright owners and it is a condition of accessing these publications that users recognise and abide by the legal requirements associated with these rights.

Take down policy

The Research Portal is Queen's institutional repository that provides access to Queen's research output. Every effort has been made to ensure that content in the Research Portal does not infringe any person's rights, or applicable UK laws. If you discover content in the Research Portal that you believe breaches copyright or violates any law, please contact openaccess@qub.ac.uk. 


\title{
An inventory of atomic species in the atmosphere of WASP-121b using UVES high-resolution spectroscopy
}

\author{
Stephanie R. Merritt ${ }^{\circledR},{ }^{1 \star}$ Neale P. Gibson ${ }^{\circledR}, 2$ Stevanus K. Nugroho ${ }^{\circledR},{ }^{1}$ Ernst J. W. de Mooij, ${ }^{1}$ \\ Matthew J. Hooton ${ }^{\oplus},{ }^{3}$ Joshua D. Lothringer, ${ }^{4}$ Shannon M. Matthews, ${ }^{1}$ Thomas Mikal-Evans ${ }^{\oplus}, 5$ \\ Nikolay Nikolov ${ }^{\oplus}{ }^{6}$ David K. Sing ${ }^{4,7}$ and Chris A. Watson ${ }^{1}$ \\ ${ }^{1}$ Astrophysics Research Centre, School of Mathematics and Physics, Queen's University Belfast, Belfast BT7 1NN, UK \\ ${ }^{2}$ School of Physics, Trinity College Dublin, Dublin 2, Ireland \\ ${ }^{3}$ Physikalisches Institut, Universität Bern, Gesellschaftsstrasse 6, CH-3012 Bern, Switzerland \\ ${ }^{4}$ Department of Physics and Astronomy, Johns Hopkins University, Baltimore, 21218 MD, USA \\ ${ }^{5}$ Kavli Institute for Astrophysics and Space Research, Massachusetts Institute of Technology, 77 Massachusetts Avenue 37-241, Cambridge, MA 02139, USA \\ ${ }^{6}$ Space Telescope Science Institute, 3700 San Martin Dr, Baltimore, MD 21218, USA \\ ${ }^{7}$ Department of Earth and Planetary Sciences, Johns Hopkins University, Baltimore, 21218 MD, USA
}

Accepted 2021 June 24. Received 2021 June 22; in original form 2020 November 2

\begin{abstract}
Ultrahot Jupiters (UHJs) present excellent targets for atmospheric characterization. Their hot dayside temperatures $(T \gtrsim 2200 \mathrm{~K})$ strongly suppress the formation of condensates, leading to clear and highly inflated atmospheres extremely conducive to transmission spectroscopy. Recent studies using optical high-resolution spectra have discovered a plethora of neutral and ionized atomic species in UHJs, placing constraints on their atmospheric structure and composition. Our recent work has presented a search for molecular features and detection of Fe I in the UHJ WASP-121b using Very Large Telescope (VLT)/UV-Visual Echelle Spectrograph (UVES) transmission spectroscopy. Here, we present a systematic search for atomic species in its atmosphere using cross-correlation methods. In a single transit, we uncover potential signals of 17 atomic species that we investigate further, categorizing five as strong detections, three as tentative detections, and nine as weak signals worthy of further exploration. We confirm previous detections of Cr I, V I, Ca I, K I, and exospheric H I and Ca II made with the High Accuracy Radial velocity Planet Searcher (HARPS) and the Echelle SPectrograph for Rocky Exoplanets and Stable Spectroscopic Observations (ESPRESSO), and independently re-recover our previous detection of Fe I at $8.8 \sigma$ using both the blue and red arms of the UVES data. We also add a novel detection of Sc II at $4.2 \sigma$. Our results further demonstrate the richness of UHJs for optical high-resolution spectroscopy.
\end{abstract}

Key words: methods: observational-techniques: spectroscopic-planets and satellites: atmospheres - planets and satellites: individual: WASP-121b.

\section{INTRODUCTION}

The recently emerging class of exoplanets known as ultrahot Jupiters (hereafter UHJs) present an intriguing subject for study and characterization. These tidally locked gas giants orbit on extremely short periods around their parent stars and thus experience extreme irradiation, increasing their dayside temperatures to the point where their chemistry and atmospheric structure are expected to differ greatly from cooler hot Jupiters (dayside $T \gtrsim 2200 \mathrm{~K}$; e.g. Parmentier et al. 2018). The increased temperature leads to the dissociation of molecules and the partial thermal ionization of atomic species in the hot dayside, thereby preventing the formation of the high-altitude cloud decks and aerosol particles that dominate the spectra of their cooler siblings (Helling et al. 2019). Instead, the dominant source of continuum opacity comes from scattering by $\mathrm{H}^{-}$ions created by the dissociation of molecular hydrogen and the abundance of

^E-mail: smerritt01@qub.ac.uk free electrons from the thermal ionization of metals (Arcangeli et al. 2018). The dissociation of molecular hydrogen at temperatures over $2500-3000 \mathrm{~K}$ also leads to atomic hydrogen becoming the dominant constituent of the atmosphere (Kitzmann et al. 2018; Lothringer, Barman \& Koskinen 2018; Parmentier et al. 2018), reducing the mean molecular weight of the atmosphere. The effects of the dissociation of molecular hydrogen, and its recombination on the cooler nightside, are also theorized to lead to increased dayside/nightside heat transport in UHJs (Bell \& Cowan 2018), further altering our expectations of the atmospheric chemistry from the assumptions made for cooler hot Jupiters.

These combined characteristics make UHJs excellent targets for atmospheric characterization via their transmission spectra (the fingerprint of the exoplanet spectrum found as the light from the host star passes through the upper layers of the atmosphere during transit). The reduced mean molecular weight of the atmosphere and high temperature increase the scale height of the atmosphere, extending the amount of atmosphere observable during transit events. The lack of high-altitude clouds and aerosols ensures that atomic and 
molecular features are less suppressed by these sources of opacity, as can often happen in cooler hot Jupiters (e.g. Gibson et al. 2013a,b; Kirk et al. 2017; Wakeford et al. 2017; May et al. 2018; Espinoza et al. 2019; Wilson et al. 2020). Additionally, when the terminator of the atmosphere is probed in transit observations, multiple temperature regimes are explored in a transition from the hot dayside to the cooler nightside (Parmentier et al. 2018), where both ionized and neutral metals/atoms and recombined molecules may be observable at the same time. Simulations of UHJs using stellar models have predicted a plethora of neutral and ionized atomic species in their atmospheres (Lothringer et al. 2018, 2020; Lothringer \& Barman 2019).

The adaptation of the high-resolution Doppler spectroscopy technique (Snellen et al. 2010), originally used to characterize spectroscopic binary systems, has provided us with a new tool to inventory the atomic and molecular constituents of exoplanet atmospheres. The large radial velocity of the planet imparts a correspondingly significant Doppler shift in its atomic and molecular lines. The planetary signal can then be separated from the telluric and stellar lines in the spectra by using detrending techniques to strip all static and quasi-static trends from the time series. The resulting residuals contain the photon noise and, hidden within, the Doppler-shifted lines from the planet's transmission spectrum. As these lines are individually resolved at high resolution, they can be extracted from the noise by cross-correlation with template spectra of the atomic or molecular species of interest, effectively summing up over hundreds or thousands of individually resolved spectral lines and strengthening the detection signal.

This method has, until recently, seen most of its success in the detection of molecules at infrared wavelengths (e.g. Brogi et al. 2012; Birkby et al. 2013; Lockwood et al. 2014). However, Doppler spectroscopy at optical wavelengths has recently played a huge part in the characterization of UHJs. This technique was used in a highly successful attempt to inventory the species present in the atmosphere of possibly the most notorious UHJ of them all, KELT-9b. The hottest known exoplanet to date with a $T_{\text {eq }}$ of $4050 \mathrm{~K}$, Hoeijmakers et al. (2018, 2019) revealed the presence of Mg I, Fe I, Fe II, Ti II, Na II, Na I, Cr II, Sc II, and Y II, along with hints of Ca I, Cr I, Co I, and Sr II. High-resolution spectroscopy has also provided detections of Fe I, Fe II, Ca I, Ca II, Mg I, Cr II, and the Balmer series of hydrogen in the UHJ KELT-20b/MASCARA-2b (Casasayas-Barris et al. 2018, 2019; Hoeijmakers et al. 2020a; Nugroho et al. 2020a; Stangret et al. 2020). High-resolution explorations of the UHJ WASP-33b revealed signs of TiO (Nugroho et al. 2017), Fe I (Nugroho et al. 2020b), and Ca II (Yan et al. 2019), though TiO was not detected in later observations by Herman et al. (2020). A search for FeH in several hot Jupiters resulted in tentative evidence for its presence in WASP-33b and KELT-20b/MASCARA-2b (Kesseli et al. 2020). An asymmetrical absorption signal of iron during transit of WASP-76b was attributed by Ehrenreich et al. (2020) to the existence of a chemical gradient.

One especially interesting target for atmospheric characterization, whether at high resolution or low, is the UHJ WASP-121b (Delrez et al. 2016). WASP-121b is in orbit around a bright ( $V=10.5) \mathrm{F} 6 \mathrm{~V}-$ type star (Høg et al. 2000), and its period of just $1.27 \mathrm{~d}$ imparts an equilibrium temperature of $2400 \mathrm{~K}$. Low-resolution observations with the Hubble Space Telescope (HST) have resulted in a detection of water in transmission, with tentative evidence for $\mathrm{TiO}$ or $\mathrm{VO}$ (Evans et al. 2016, 2018; Tsiaras et al. 2018); water was also detected in emission, leading to the first direct measurement of a temperature inversion (Evans et al. 2017). This temperature inversion was later confirmed by phase-curve photometry from the Transiting Exoplanet Survey Satellite (TESS; Bourrier et al. 2020b; Daylan et al. 2021). New secondary eclipse measurements made by HST recently reconfirmed the presence of water emission features (Mikal-Evans et al. 2020), though these observations have failed to reproduce a feature in the emission spectrum previously attributed to VO in Evans et al. (2017) and Mikal-Evans et al. (2019), since attributed to systematics. Recent ultraviolet (UV) observations taken during transit, also with $H S T$, discovered Fe II and $\mathrm{Mg}$ II features extending far higher in the atmosphere than previously detected features at redder wavelengths, evidence that WASP-121b has an extended, escaping atmosphere (Sing et al. 2019). A similar excess in UV absorption had previously been detected by Salz et al. (2019). Eclipse observations taken in the $z^{\prime}$ band by Mallonn et al. (2019) placed upper limits on WASP-121b's albedo, and potential variability in its atmosphere has been posited based on Gemini/Gemini Multi-Object Spectrograph (GMOS) observations (Wilson et al. 2021).

At high resolution, a search for $\mathrm{TiO}$ or $\mathrm{VO}$ in the transmission spectrum of WASP-121b using the UV-Visual Echelle Spectrograph (UVES) on the Very Large Telescope (VLT) failed to detect either (Merritt et al. 2020), in conflict with earlier low-resolution work (Evans et al. 2016, 2018; Tsiaras et al. 2018), though the inaccuracy of the VO high-temperature line list was cited as a possible cause for the non-detection of VO. Analysis by Gibson et al. (2020) of the same UVES transits, and by Bourrier et al. (2020a) and Cabot et al. (2020) using the High Accuracy Radial velocity Planet Searcher (HARPS) on La Silla, have detected Fe I deeper in the atmosphere, with Cabot et al. (2020) additionally detecting the presence of $\mathrm{H} \alpha$. Analysis of the same HARPS data by Ben-Yami et al. (2020) confirmed the presence of Fe I and Fe II, while presenting additional detections of VI and CrI and a non-detection of TiI. Further analysis by Hoeijmakers et al. (2020b), again on the same HARPS observations, confirmed the presence of Fe I, V I, and CrI, while adding detections of $\mathrm{Mg}$ I, Na I, Ca I, and Ni I, and confirming non-detections of Ti I and TiO. Finally, a study by Borsa et al. (2021) using data from the Echelle SPectrograph for Rocky Exoplanets and Stable Spectroscopic Observations (ESPRESSO)/VLT confirmed many of the previous detections and added novel detections of $\mathrm{K}$, Li I, and exospheric Ca II. This wealth of information from only a handful of data sets showcases the huge potential of high-resolution spectroscopy as a tool to explore the atmospheres of UHJs.

In order to confirm and expand upon previous detections, we present an atomic species inventory of the UHJ WASP-121b, using high-resolution spectra taken with VLT/UVES (Dekker et al. 2000), an instrument which has seen successful use for the exploration of exoplanet atmospheres (Snellen 2004; Khalafinejad et al. 2017; Gibson et al. 2019). The blue arm of these observations was previously explored by Gibson et al. (2020), and analysis of the red arm in a search for molecular features was presented in Merritt et al. (2020): this paper presents analysis of both the red and blue arms combined with the aim of searching for a broad range of species. In Section 2, we describe the observations and the extraction of the spectra. Section 3 discusses our data-processing steps, the creation of model transmission spectra for cross-correlation, the cross-correlation process, and our injection tests. Results of a broadscale atomic species search are presented alongside our detection criteria in Section 4; the results are further discussed in detail in Section 5, including the results of our injection tests and notable non-detections.

\section{OBSERVATIONS AND DATA REDUCTION}

Two transits of WASP-121b were observed using the high-resolution echelle spectrograph UVES, mounted on the 8.2-m 'Kueyen' telescope (UT2) of the VLT. Observations were taken on the nights of 
2016 December 25 and 2017 January 4 as part of program 098.C0547 (PI: Gibson). The second transit was discarded due to low signal-to-noise ratio $(\mathrm{S} / \mathrm{N})$ caused by a loss in guiding. The peak $\mathrm{S} / \mathrm{N}$ over the course of the observations can be found in Merritt et al. (2020). The blue arm of this data was previously presented in Gibson et al. (2020), and the red arm of this data was previously presented in Merritt et al. (2020): further details on the observations can be found there. The subsequent analysis in this paper uses both arms for a total of 64 spectral orders from 375 to $866 \mathrm{~nm}$.

Extraction proceeded almost identically to the process outlined in Merritt et al. (2020), using the custom PYTHON pipeline outlined in that work. However, bias subtraction was found to lead to spuriously negative values in low-flux areas of the spectrum in the blue arm, and a correction from the overscan areas was implemented. Additionally, the order traces from the ESOREX pipeline were found to be slightly off-centre, and were corrected. Once again, the five spectral traces filled the extraction aperture almost completely, so no background subtraction could be performed. Calculation of the Barycentric Julian Date $\mathrm{BJD}_{\mathrm{TDB}}$ for each observation was performed using functions from the ASTROPY.TIME PYTHON package.

Outliers were removed from the spectra by a two-stage process slightly improved from the method outlined in Merritt et al. (2020). First, the spectra were $\sigma$ clipped for values over $3 \sigma$, which were replaced by the median value of the surrounding 100 pixels. As this did not remove all outliers, a single-component principal component analysis (PCA) reconstruction of the time-series spectra then was subtracted from the spectra to remove all time- and wavelengthdependent trends. Two iterations of $\sigma$-clipping were performed upon the residuals, with the values of any outliers set to zero: adding the PCA reconstruction back to the data then replaced the clipped outliers with their value from the PCA reconstruction. Although a $3 \sigma$ clipping might appear aggressive, it replaced an average of only 0.07 per cent of pixels per order, and does not impact our results.

In an additional divergence from Merritt et al. (2020), spectral alignment was performed to correct for small shifts in the original wavelength solution provided by the ESOREX pipeline. This was not thought to be necessary in the search for TiO and VO presented in Merritt et al. (2020), as the total wavelength drift over each night was found to be significantly less than a resolution element $\left(\sim 2.5 \mathrm{~km} \mathrm{~s}^{-1}\right.$ at $R \sim 110000$ ). However, Gibson et al. (2020) found that orderto-order drift in the blue arm was $\sim 3 \mathrm{~km} \mathrm{~s}^{-1}$, probably due to inaccuracies in the wavelength solution provided by the pipeline. Additionally, as the goal of this work is to search for atomic species, many of which may be present in the stellar spectrum, the need for an accurate wavelength solution is increased in order to optimize the detrending methods used to remove the stellar spectrum from the data, as outlined in Section 3.1.

The blue arm of the spectra was aligned via cross-correlation with a PHOENIX model for an F6V-type star (Husser et al. 2013). Though this would also fit for the Rossiter-McLaughlin (RM) effect, WASP-121b is on a near-polar orbit with a projected obliquity of $258^{\circ}$ (Delrez et al. 2016), and the RM effect is expected to have an amplitude well below both the change in velocity of WASP-121b during transit and the resolution limit of UVES (Gibson et al. 2020). The RM effect is therefore expected to have a negligible effect on both alignment and on species detection, with the latter confirmed via modelling by Cabot et al. (2020).

The resultant detected velocity shifts were smoothed with a highpass filter and applied to each frame (via linear interpolation) and order of the blue arm data separately. In the red arm, alignment could not be successfully performed to the stellar spectrum due to the lack of lines and the contamination due to the presence of tellurics.
Instead, alignment was performed via cross-correlation to a telluric template generated from ESO's SKYCALC tool (Noll et al. 2012; Jones et al. 2013). In the red arm, the order-to-order discrepancy in the wavelength solution was found to be much less $\left(\sim 1 \mathrm{~km} \mathrm{~s}^{-1}\right)$, and due to the sporadic placement of tellurics throughout the red arm spectra, order-specific alignment was very difficult for orders with few tellurics. As a result, the wavelength corrections for each frame were averaged over the orders and applied on a frame-by-frame basis only.

We note that in the process used to correct the wavelength solution, the spectra in the blue arm are aligned to the stellar rest frame. This simultaneously corrects for the time-dependent barycentric velocity variations and the systemic velocity of WASP-121. As the spectra in the red arms are aligned to the telluric rest frame they are not yet corrected for these effects. We later realign the red arm to the stellar rest frame; however, as the tellurics dominate the systematics in the red arm (which is also the reason why telluric features were used for the alignment), we first perform systematic corrections before performing the realignment. This is discussed in Section 3.1. Simultaneously with the correction of the wavelength solution, the spectra were also linearized in wavelength and supersampled to $2 / 3$ of the average pixel width for each order. Minimizing the number of interpolations performed on the data helps preserve spectral information and reduces the introduction of noise. The results of the extraction were cross-checked with an independent pipeline (Gibson et al. 2020).

\section{ANALYSIS}

\subsection{Pre-processing}

We follow an almost identical methodology to that outlined in Merritt et al. (2020), based on techniques often used in searches for molecular and atomic species in high-resolution spectra (e.g. Snellen et al. 2010; Brogi et al. 2012; Birkby et al. 2013; Nugroho et al. 2020a). In order to search for the buried exoplanetary spectral signal, we must first remove all other trends from the time series that are static or quasi-static in time, including the stellar spectrum and telluric absorption from the Earth's atmosphere, further modulated by the (time-varying) response of the instrument. The removal of these trends should, in theory, results in residuals that are composed of purely photon noise and the Doppler-shifted, continuum-removed planetary signal. In practice however, the pre-processing is imperfect, and neither perfectly removes the stellar and telluric lines, nor leaves the atmospheric signature of the planet untouched. Here, we briefly recap the data processing, and an example on a single spectral order is shown in Fig. 1.

First, we place all of the spectra on a 'common' blaze function, which can vary with time. The correction is performed as in Merritt et al. (2020) by dividing each order of time-series spectra by the median spectrum, smoothing the resulting residual spectra with a median filter with a width of 15 pixels and a Gaussian filter with a standard deviation of 50 pixels, and dividing through each by its smoothed residual spectra to correct the blaze variation.

Next, all static and quasi-static trends in time - such as the 'common' blaze function, the stellar spectrum, and the telluric spectrum - are removed from the spectral time series using the SYSREM algorithm (Tamuz, Mazeh \& Zucker 2005), a tool in standard use for the detrending of high-resolution time-series data (e.g. Birkby et al. 2013, 2017; Nugroho et al. 2020a). One advantage SYSREM has over other PCA-based detrending algorithms is its inherent treatment of the pixel uncertainties. Unlike Merritt et al. (2020), we use the 

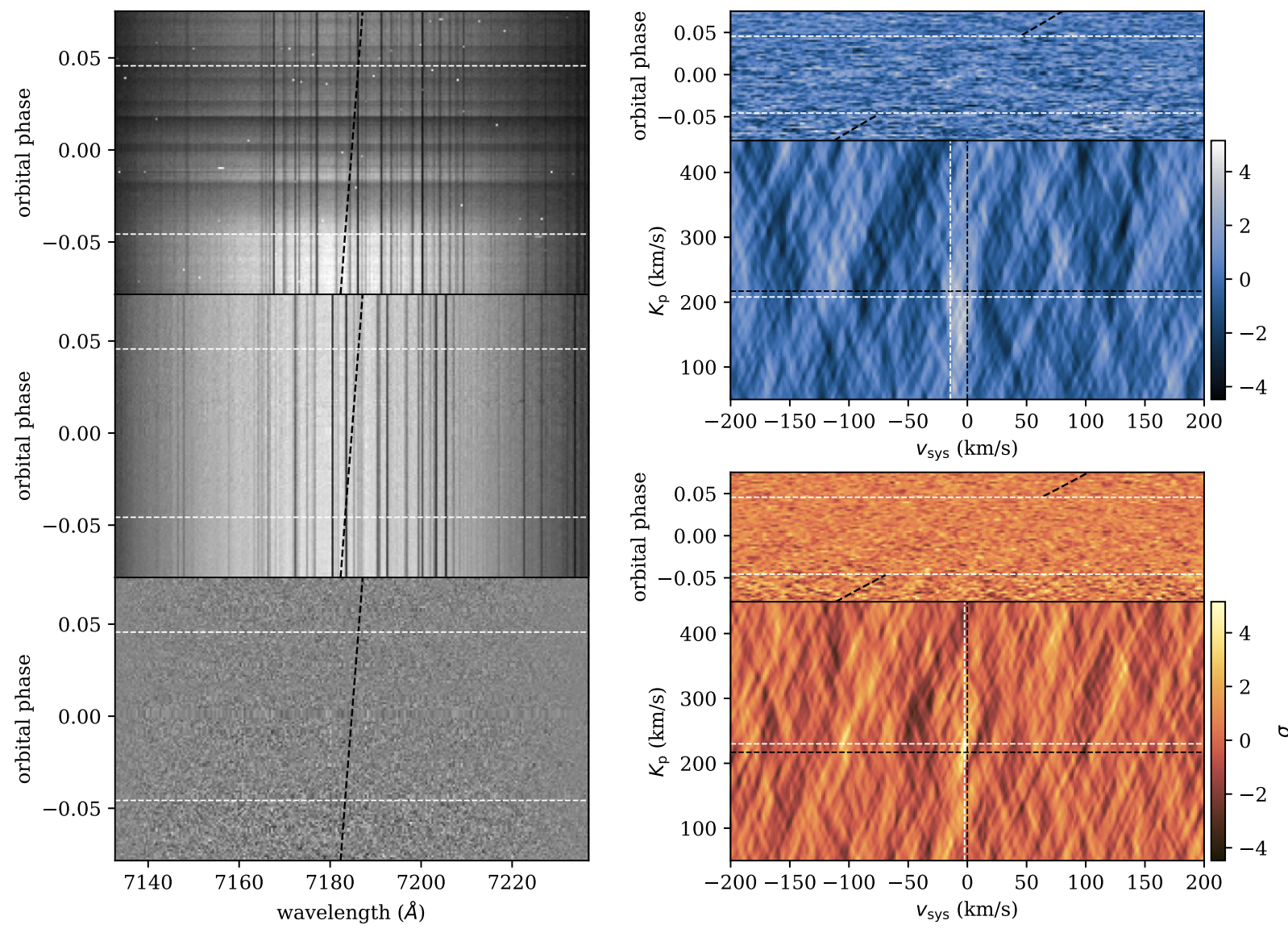

Figure 1. Pre-processing and cross-correlation. Left: data processing of a single raw echelle order (top), after wavelength alignment and correction for blaze variation (middle), and after division by SYSREM model and weighting by uncertainties (bottom). The dashed white lines indicate the times of ingress and egress. The dashed black line indicates the approximate velocity shift of WASP-121b. Right: the summed cross-correlation function (CCF) time series (upper) and cross-correlation velocity map (lower) for the blue (top) and red (bottom) arms of the data, respectively, for the Cr I model used to retrieve the signal presented in Fig. 3. The white dashed lines in the CCF time series plots represent the times of ingress and egress. The black dashed lines indicate the expected position of the signal 'trace' in the CCF time series. In the velocity maps, the black dashed cross-hairs indicate the expected position of the signal; the white dashed cross-hairs mark the peak of the detected signal.

Poisson pixel uncertainties calculated from the extracted spectra, which resulted in more efficient removal of the stellar signal than the outer-product variance method described in that work.

The blaze correction was found to be unstable at the bluest end of each spectral order, an effect seen in both arms of the data. We thus remove the first 900 and last 90 pixels of each order in the blue arm (identically to Gibson et al. 2020 after accounting for 3/2 supersampling) and the first 750 pixels of the red arm (identically to Merritt et al. 2020, likewise). This removes 22 per cent of the pixels in the blue arm spectra and 12 per cent in the red arm. While this is a significant chunk of the data, the edges of the orders are low in $\mathrm{S} / \mathrm{N}$, therefore this has minimal impact on our detections.

We run multiple passes of SYSREM to create a $2 \mathrm{D}$ model representation for each time series. Instead of using the resulting model-subtracted residuals as our final product, we instead sum the SYSREM models for each pass and divide our time-series spectra by this model before subtracting 1 . This process preserves the relative line strengths of the planet's transmission spectrum, without first having to divide through by the stellar spectrum (Gibson et al. 2020). The data uncertainties are also divided through by the model. The detrended residuals are then optimally weighted by the square of the pixel Poisson uncertainties.
In the blue arm, where stellar lines dominate, SYSREM is performed in the rest frame of the star. In the red arm, SYSREM is performed in the telluric rest frame, in order to optimize the removal of the dominant telluric spectrum. Thus, after SYSREM detrending, the red arm is then further corrected for the systemic and barycentric velocities as outlined in Section 2, to bring the red arm spectra into the stellar rest frame. We note that the barycentric correction and systemic velocity are known to much higher precision than the instrument resolution, and therefore the different methods used to align the spectra in the red and blue arms will not impact our results.

\subsection{Model transmission spectra}

Detecting atomic features using the cross-correlation technique requires spectral models of each species. To create these models, high-temperature line lists of the species of interest are used to generate absorption cross-sections. These cross-sections are then incorporated into radiative transfer models, which generate model transmission spectra for cross-correlation.

Cross-sections for neutral atomic species and their singly ionized counterparts numbering from atomic number 1 - hydrogen - to atomic number 39 - yttrium - were generated following Nugroho 
et al. (2017, 2020a,b) using HELIOS-K (Grimm \& Heng 2015) with the Kurucz atomic line lists (Kurucz 2018). We used a Voigt line profile with thermal and natural broadening only at a resolution of $0.01 \mathrm{~cm}^{-1}$ with an absolute line wing cut-off of $1 \times 10^{7} \mathrm{~cm}^{-1}$, except for $\mathrm{HI}$ and $\mathrm{He} \mathrm{I}$ that used $3 \times 10^{4}$ and $1 \times 10^{5} \mathrm{~cm}^{-1}$, respectively. These values were chosen to ensure important lines are not washed away. We chose to explore no further than yttrium (as beyond this point, solar abundances become very low) with the exception of Cs I, due to its presence in late-M and L-dwarfs (e.g. Basri et al. 2000).

Rather than producing a transmission spectrum for each species via numerical integration over a layered atmosphere, we instead elect to use the analytical equation for an approximate transmission spectrum used in Gibson et al. (2020), adapted from the work of Heng \& Kitzmann (2017). This equation computes the effective radius as

$R(\lambda)=R_{0}+H\left[\gamma+\ln \left(\frac{P_{0}}{m g} \sqrt{\frac{2 \pi R_{0}}{H}}\right)\right]+H \ln \sum_{j} \chi_{j} \sigma_{j}(\lambda)$.

Here, $H, g$, and $m$ are the scale height, surface gravity, and mean molecular mass of the atmosphere, $R_{0}$ and $P_{0}$ are the reference radius and pressure, $\gamma=0.56$ is a dimensionless constant, $\chi_{j}$ is the volume mixing ratio of species $j$, and $\sigma_{j}$ is the cross-section of species $j$. Scattering is accounted for by including Rayleigh scattering using the cross-section of Lecavelier Des Etangs et al. (2008) and a grey cloud deck simulated by simply truncating the model at a given pressure level $P_{\text {cloud }}$. We disregard the important effects of $\mathrm{H}^{-}$scattering (Parmentier et al. 2018) in order to avoid any further degeneracy with Rayleigh scattering and the cloud deck.

Gibson et al. (2020) found this to produce models of sufficient accuracy for high-resolution detections, by comparing directly with a full radiative transfer model, as long as the same common assumptions of no pressure broadening, a well-mixed atmosphere, and an isothermal atmosphere are made. The main benefit of this analytical equation lies in its speed, which allows us to swiftly create hundreds of spectra for a range of parameters. This greatly increases the efficiency of a broad species search and allows quick exploration of the effects of changing parameters with minimal loss in the accuracy of the template.

We generated spectra for every species at three temperatures of 2000,2500 , and $3000 \mathrm{~K}$ based on the most recent estimates of the temperature of WASP-121b at the limb (Mikal-Evans et al. 2019; Gibson et al. 2020). We additionally generated models using a higher set of temperatures (from 3500 to $9500 \mathrm{~K}$, in steps of $500 \mathrm{~K}$ ) for the seven ions for which we found signals (given in Section 4). We assume a Jupiter-like composition for the mean molecular mass, and the scale height, which is expected to vary with temperature, was instead fixed at $H=960 \mathrm{~km}$ using a temperature of $2800 \mathrm{~K}$ (approximately the temperature at the top of the atmosphere as measured by Mikal-Evans et al. 2019). As we are subtracting the continuum from both the data (as outlined in Section 3.1) and the model spectra themselves, we are not particularly sensitive to the scale height as a parameter: changing the scale height simply scales the model amplitude, which should not affect the results of the crosscorrelation detections (although does impact the injection tests). The reference radius and pressure were set at $1.8 R_{\mathrm{J}}$ and $20 \mathrm{mbar}$, respectively. A full list of system and planetary parameters used in this work is presented in Table 1.

Instead of varying the volume mixing ratio or VMR of each species, as was done in Merritt et al. (2020), we choose to vary the position of the grey cloud deck, $P_{\text {cloud }}$. Four values of $P_{\text {cloud }}$ were chosen (1 bar, 0.05 bar, 1 mbar, and 0.05 mbar). These were not physically motivated but instead selected to vary the amount
Table 1. Stellar and planetary parameters for the WASP-121b system utilized in this paper.

\begin{tabular}{|c|c|}
\hline Parameter & Value \\
\hline \multicolumn{2}{|l|}{ WASP-121 } \\
\hline$M_{\star}\left(\mathrm{M}_{\odot}\right)$ & $1.353_{-0.079}^{+0.080 a}$ \\
\hline$R_{\star}\left(\mathrm{R}_{\odot}\right)$ & $1.458 \pm 0.080^{a}$ \\
\hline Spectral type & $\mathrm{F}^{6} \mathrm{~V}^{a}$ \\
\hline$T_{\text {eff }}(\mathrm{K})$ & $6459 \pm 140^{a}$ \\
\hline$V$-magnitude & $10.44^{a}$ \\
\hline$v_{\text {sys }}\left(\mathrm{km} \mathrm{s}^{-1}\right)$ & $38.36 \pm 0.43^{b}$ \\
\hline \multicolumn{2}{|l|}{ WASP-121b } \\
\hline$T_{0}\left(\mathrm{BJD}_{(\mathrm{TDB})}\right)$ & $2457599.551478 \pm 0.000049^{c}$ \\
\hline$P(\mathrm{~d})$ & $1.2749247646 \pm 0.0000000714^{c}$ \\
\hline$a / R_{\star}$ & $3.86 \pm 0.02^{d}$ \\
\hline$R_{\mathrm{p}} / R_{\star}$ & $0.1218 \pm 0.0004^{d}$ \\
\hline$i\left(^{\circ}\right)$ & $89.1 \pm 0.5^{d}$ \\
\hline$T_{\mathrm{eq}}(\mathrm{K})$ & $>2400$ \\
\hline$H(\mathrm{~km})$ & $\sim 960^{d}$ \\
\hline$K_{\mathrm{p}}\left(\mathrm{km} \mathrm{s}^{-1}\right)$ & $\sim 217$ \\
\hline
\end{tabular}

Notes. ${ }^{a}$ Adopted from Delrez et al. (2016). ${ }^{b}$ Adopted from Gaia Collaboration (2016, 2018). ${ }^{c}$ Adopted from Sing et al. (2019). ${ }^{d}$ Adopted from Evans et al. (2018).

of truncation of each model, to give a good range of lines present above the continuum, for an arbitrarily chosen VMR of $10^{-8}$. An example of the 12 models created for a single species ( $\mathrm{Cr} \mathrm{I}$ ) is shown in Fig. 2. Given the degeneracy between reference pressure, radius, and abundance (e.g. Benneke \& Seager 2012; Heng \& Kitzmann 2017), this approach is not sensitive to the abundances. Instead, this forces us to consider detections entirely in the sense of line strength present above the continuum (the only parameter we can meaningfully measure with our methodology).

This process results in 1312 model spectra being generated: three temperatures and four values of $P_{\text {cloud }}$ for 40 neutral species and 37 singly ionized forms, plus an additional 13 temperatures for seven of the ionized species. However, we then eliminate any models with no or extremely weak lines in the wavelength ranges of the blue and red arms of our data, reducing the number to 792 models covering 43 neutral and ionized species. We did not constrain our model selection in any other way, such as by the supposed detectability of the species, as such constraints are invariably model dependent.

Finally, before cross-correlation, the continuum and scattering profile is removed from all of the model spectra, as the pre-processing steps outlined in Section 3.1 remove all large-scale variation from our data, including from any extant exoplanetary signal. This continuum removal is performed by subtracting a low-pass filter consisting of a 1400-pixel maximum filter applied in the wavelength direction that is subsequently smoothed by a Gaussian filter with a kernel of 400 pixels, removing all large-scale variations in the model and normalizing it at zero.

\subsection{Cross-correlation}

As described in Section 3.1, we divide the spectra (and uncertainties) by a SYSREM model in order to remove all dominant trends before weighting the residuals by the pixel variance. We create three sets of weighted SYSREM residuals for each arm, using 10, 15, and 20 passes of SYSREM. Currently there exists no reliable method of determining the optimal number of SYSREM iterations beyond empirically optimizing the strength of a recovered signal or injection. Though this method was used in Merritt et al. (2020), it is not 


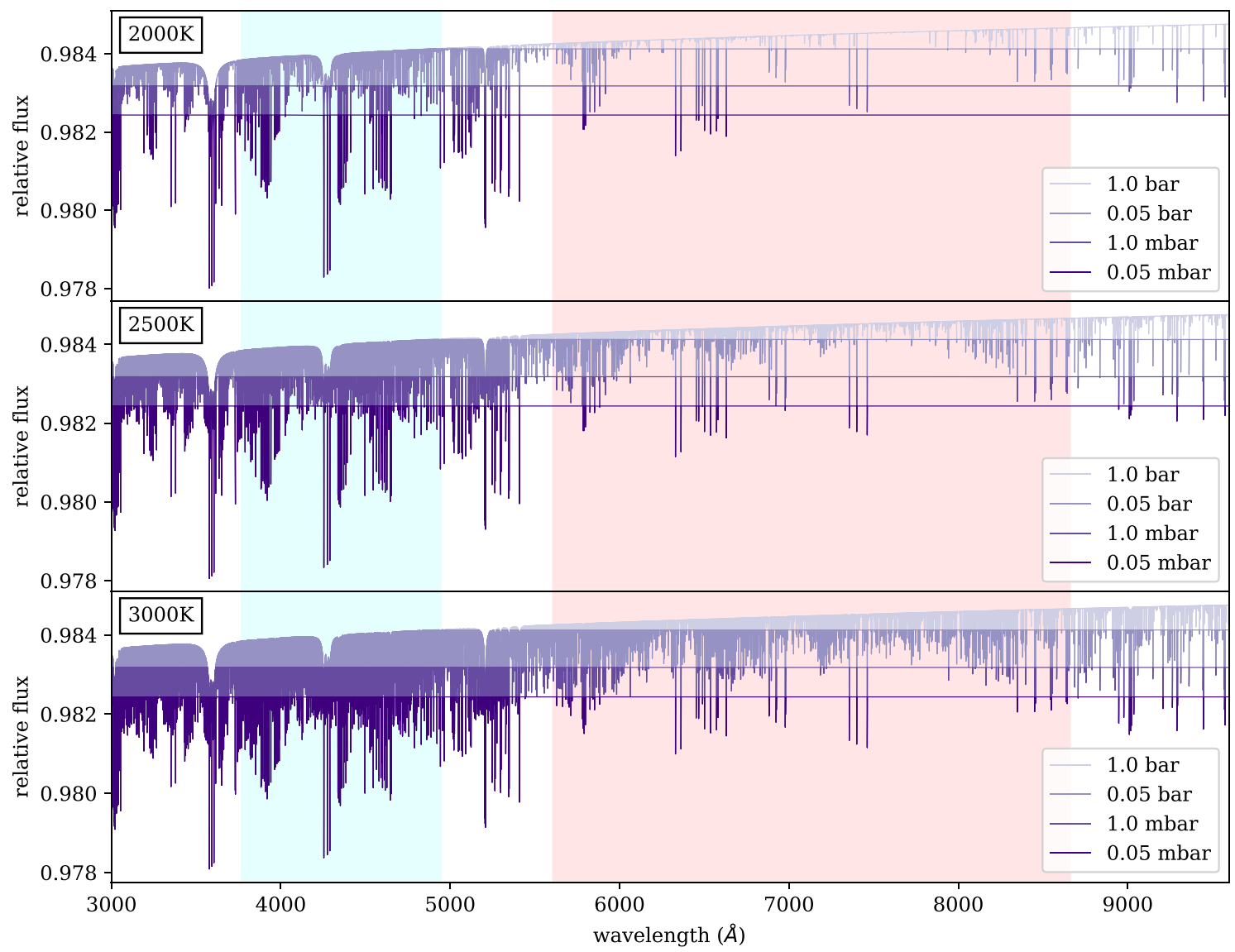

Figure 2. Examples of the model transmission spectra for Cr I in WASP-121b at three different temperatures and four different positions of the grey cloud deck $P_{\text {cloud }}$. The log volume mixing ratio is held at $10^{-8}$. The blue and red regions indicate the blue and red wavelength ranges of the UVES data.

particularly feasible here given our search for a multitude of different species, and we instead choose to test a small range. As many of the species searched for are expected to exist in the stellar spectrum, this range encompasses a higher number of SYSREM iterations than the nine used in Merritt et al. (2020) in order to maximize removal of stellar residuals.

We then cross-correlate each frame of each order with the corresponding section of the model spectra, binned down to the instrumental resolution of the order. As described in Merritt et al. (2020), no planetary signal is expected in the out-of-transit frames. A basic transit model was thus generated using the equations of Mandel \& Agol (2002) (assuming no limb darkening), and this was used to weight the time series of cross-correlation functions (CCFs).

A peak is seen in the CCF where a match is found between the template spectrum and the spectral residuals. Where such a peak is present, it will be Doppler shifted according to the motion of the planet around the parent star. This results in a diagonal trace in the cross-correlation time series that follows the Doppler shift over time. This trace can often be seen with the naked eye in cases where the signal is strong (e.g. Snellen et al. 2010). To enhance weaker signals, the cross-correlation time series is integrated over a range of potential radial velocity curves. The radial velocity $v_{\mathrm{p}}$ at each frame of the observations is given by

$v_{\mathrm{p}}(\phi)=v_{\mathrm{sys}}+K_{\mathrm{p}} \sin (2 \pi \phi)$

where $v_{\text {sys }}$ is the systemic velocity, $K_{\mathrm{p}}$ is the radial velocity semiamplitude of the planet, and $\phi$ is the orbital phase of the planet at the time of observation, where $\phi=0$ represents the mid-transit time. As the spectra have already been corrected to the stellar rest frame, $v_{\text {sys }}$ is expected to be zero. The $x$-axis of the cross-correlation time series can therefore be considered to represent the difference in velocity or 'lag' - from the stellar rest frame.

The cross-correlation time-series is then integrated over a range of potential values of $K_{\mathrm{p}}$ by linearly interpolating each order to a radial velocity curve generated using values for $K_{\mathrm{p}}$ from 100 to $400 \mathrm{~km} \mathrm{~s}^{-1}$ in steps of $1 \mathrm{~km} \mathrm{~s}^{-1}$. This step size is smaller than the average single resolution element of the original spectra $\left(\sim 3.15 \mathrm{~km} \mathrm{~s}^{-1}\right.$ averaged over both arms). A smaller range than in Merritt et al. (2020) was chosen in order to speed up computational time, while still remaining large enough to judge the overall noise profile of the resultant map. For a subset of species, we extended the $K_{\mathrm{p}}$ range to check for artefacts around $K_{\mathrm{p}}=0$ and $v_{\text {sys }}=0$, which could arise from poorly subtracted systematic effects. However, we found no such evidence.

Each 2D order of $K_{\mathrm{p}}$-interpolated CCFs is then summed over the columnwise time direction. These 1D sums are stacked in $K_{\mathrm{p}}$ order, creating one 2D velocity heat map for each spectral order. The $y$-axis of these heat maps is $K_{\mathrm{p}}$; the $x$-axis $v_{\text {sys }}$ represents, as before, the velocity difference from the stellar rest frame. Examples of the CCF time series and the resulting velocity maps are shown for both the red and blue arms in the right-hand column of Fig. 1.

The $v_{\text {sys }}$ scale of the CCFs, and thus the velocity heat maps, is set by the wavelength scale of the spectral order. Therefore, due to the varying resolution across the spectral range, the map for each order has a slightly different scale in $v_{\text {sys. }}$. The maps for the blue and red 
arms are thus linearly interpolated to a velocity scale made up of the mean of the $v_{\text {sys }}$ scales for that arm and summed over the orders to form a final map for the blue and the red arm. The individual order maps are also combined into a final red+blue map by interpolating to the mean of all $v_{\text {sys }}$ scales and summing again over the orders, which have already been optimally weighted by their variance: this should have the effect of strengthening extant signals by using the full spectral range of our data.

Because of our earlier removal of the continuum from the data, and the subsequent use of cross-correlation, the $z$-axis of the velocity heat maps is in arbitrary units. To set a detection significance we initially used the common method also used in Merritt et al. (2020). An area from -50 to $50 \mathrm{~km} \mathrm{~s}^{-1}$ in $v_{\text {sys }}$ is masked in order to exclude any potential signal, and the standard deviation of the rest of the map calculated. The full unmasked map is then divided by this standard deviation.

Again similarly to Merritt et al. (2020), we also attempted obtaining detection significances using the more robust phase-shuffling method, as seen in Esteves et al. (2017). The time series of CCFs is linearly interpolated to a common $v_{\text {sys }}$ scale and summed over the orders to form a 'master' set of CCFs, which is then shuffled randomly in time before regenerating the cross-correlation maps. This is performed 1000 times, and the standard deviation at each pixel of the maps found, creating a standard deviation map by which the original, unshuffled map was divided. This method was only used on a limited subset of the maps (those in which potential detections were found) due to the computationally intensive nature of the method. Also, as in Merritt et al. (2020), we found no discernible difference using this method beyond a slight increase in detection significance for most signals. Once again, we prefer to use the method that generates a more conservative estimate of detection significance. Detection significance was, in all cases, set independently for the red, blue, and red+blue maps.

The fully automated cross-correlation process was performed for every atomic model template outlined in Section 3.2, with three cross-correlation maps (the blue arm, the red arm, and the combined red+blue map) generated and plotted for each model. The automated signal detection looked for the strongest peak within a range of 10 $\mathrm{km} \mathrm{s}^{-1}$ in $v_{\text {sys }}$ and $20 \mathrm{~km} \mathrm{~s}^{-1}$ in $K_{\mathrm{p}}$ from the 'expected' signal position of $217 \mathrm{~km} \mathrm{~s}^{-1}$ (from parameters in Delrez et al. 2016) and $0 \mathrm{~km} \mathrm{~s}^{-1}$ (i.e. the stellar rest frame). Peaks in the maps of over $3 \sigma$ in this range were highlighted for further inspection, although in practice every cross-correlation map was investigated by eye for potential signals. Uncertainties were estimated for the measurements of $v_{\text {sys }}$ and $K_{\mathrm{p}}$ by taking the full width at half-maximum (FWHM) of the signal in the relevant axis on the map and converting this to a standard deviation. This in effect assumes that the cross-correlation signal is proportional to the likelihood function, and that the uncertainties are uncorrelated with other parameters. A more detailed treatment of the uncertainties in the velocity constraints will be left to future work.

\subsection{Injection tests}

In order to test our sensitivity to the species we are searching for, and to judge the reliability of any potential signals, we also performed injection tests for every model used in the signal retrieval process. Every model was convolved with the instrumental resolution using a top-hat function and Doppler shifted to conform to a $K_{\mathrm{p}}$ curve of $-217 \mathrm{~km} \mathrm{~s}^{-1}$. This value is the negative of the expected $K_{\mathrm{p}}$, and was selected as it leads to a reversed but otherwise identical velocity curve to the expected signal, thus ensuring maximum similarity to a real signal while also ensuring that the injection is not artificially boosted by an extant signal. We also take into account the fact that the red arm spectra are not corrected to the stellar rest frame until after SYSREM detrending by introducing a 'decorrection' for barycentric and systemic velocity into the red-arm injected models. The Dopplershifted models are then multiplied into the original extracted spectra before the pre-processing steps outlined in Section 3.1, after which the cross-correlation process continues almost identically. However, injection tests were performed with 15 passes of SYSREM exclusively, due to the computationally intensive nature of the process.

We also performed additional injection tests for all ionized species that showed signals in detection. The models generated in Section 3.2 assume hydrostatic equilibrium, but from Sing et al. (2019) we are aware that WASP-121b is likely to have an escaping exosphere containing ionized species, resulting in much deeper spectral features than would be expected from our simple model. These injection tests used models generated in an identical manner to those described in Section 3.2 for the higher temperature range of 3500-9500 K. These models were then scaled so that their features matched the rough height of $\sim 0.25-0.3 R_{\mathrm{p}} / R_{\star}$ reported for Fe II and $\mathrm{Mg}$ II in Sing et al. (2019). For similar reasons, we also scaled the H I models so that the $\mathrm{H} \alpha$ line matched the height of $\sim 0.2 R_{\mathrm{p}} / R_{\star}$ reported by Cabot et al. (2020)

\section{RESULTS}

\subsection{Detection criteria}

The cross-correlation process resulted in a signal presents within the detection range for 17 different species, with a huge range in detection strength, signal position, and level of noise/additional structure in the cross-correlation map. Additionally, a further three signals were found outside of our detection range that are none the less of interest.

Simply applying a detection significance cut-off of $4 \sigma$, similarly to Merritt et al. (2020), was found to be insufficient as a means of judging the reliability of the potential signals. For example, some otherwise-dubious signals were found to be stronger than $4 \sigma$, while some more likely signals fell just below. In addition, the methods used to generate the detection significances are, though common in the literature, rather simplistic, and rely on an assumption of Gaussian white-noise statistics that largely ignores the presence of structure in the map. In reality, the statistics of these cross-correlation maps are poorly understood, and the detection significance can be changed by as much as $0.5 \sigma$ by simply changing the area used to calculate the standard deviation, or by increasing/decreasing the number of phase-shuffling iterations. New techniques are being pursued and have produced encouraging results (Brogi \& Line 2019; Fisher et al. 2020; Gibson et al. 2020; Nugroho et al. 2020b), but due to their computationally intensive nature, these methods were not attempted here for our large range of species.

As a result, we categorize our prospective detections using a more extensive set of diagnostic criteria. The intention of these criteria is primarily to rule out or weaken confidence in signals that may initially seem strong but are problematic in other areas. Signals must fulfil the majority of these criteria to be considered as detections. The initial set of 17 species was selected via examination by eye of all of the resulting cross-correlation maps. We then judged them considering the following criteria.

(i) Detection strength at or above $3.5 \sigma$. Though it has been shown (e.g. by Cabot et al. 2019) that noise fluctuations in the cross-correlation map can reach the $4 \sigma$ level, we use a slightly less conservative value to avoid missing potentially interesting signals. 

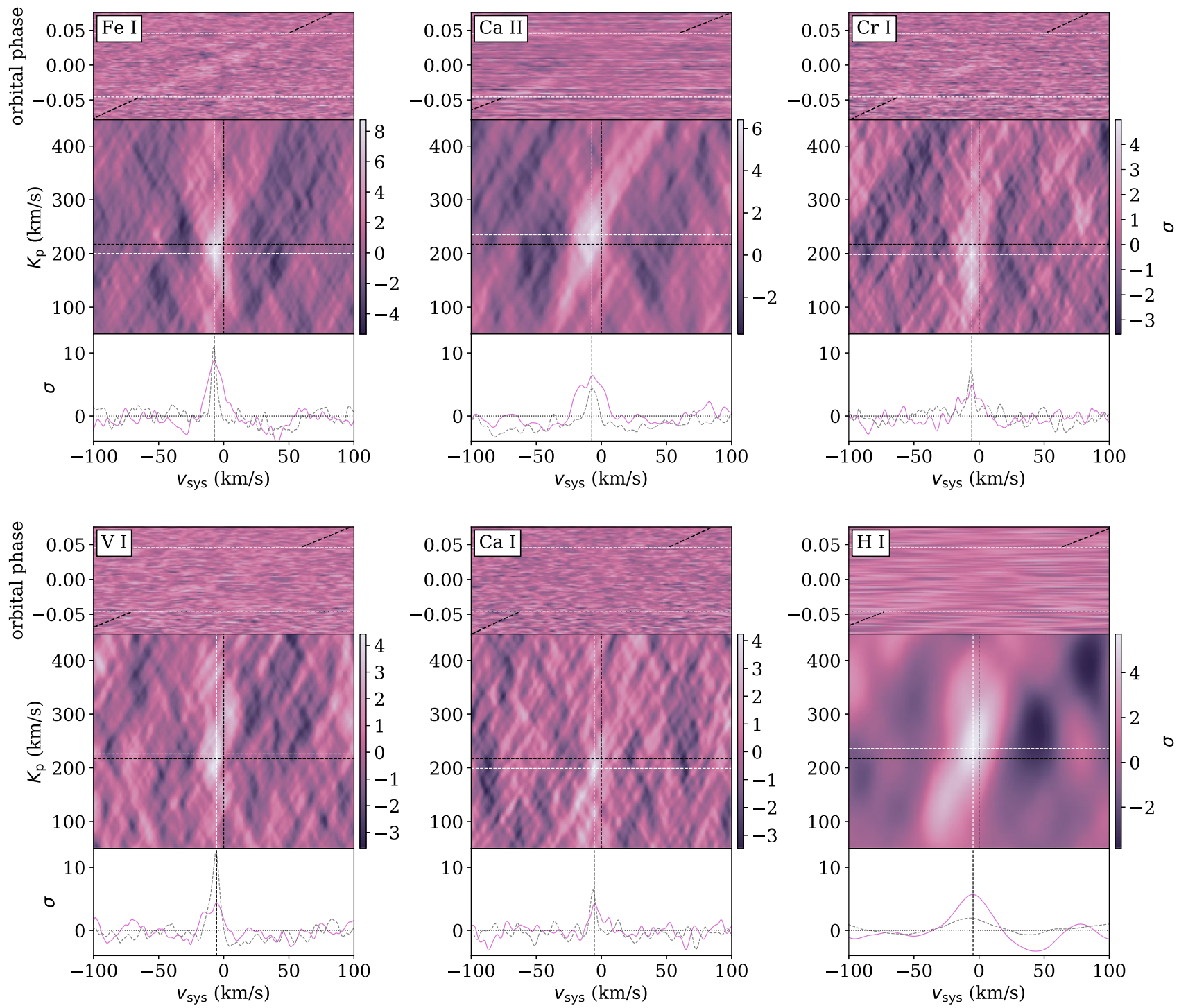

Figure 3. Results from cross-correlation for Fe I, Ca II, Cr I, V I, Ca I, and H I. Top: the summed cross-correlation functions (CCFs) for the blue and red arms. The dashed white lines indicate ingress and egress. The dashed black line indicates the expected position of the signal trace. Middle: the velocity map. The black dashed cross-hairs indicate the expected position of the signal, while the white cross-hairs centre on the peak of the detected signal. Bottom: a slice of the velocity map at the value of $K_{\mathrm{p}}$ for which the signal was located. The purple line is from the velocity map presented: the dotted grey line shows the signal from the injection test of the same model, adjusted to have the same $v_{\text {sys }}$ offset as the detected signal. The smoother CCFs and map seen for H I are the result of the template spectrum being dominated by a few broad lines. The results for Ca II are presented in further detail in Fig. 4.

Any signals found below this cut-off were generally disregarded, with three potentially interesting exceptions.

(ii) Position of the signal. Gibson et al. (2020) recovered a strong Fe I signal at a $v_{\text {sys }}$ offset of $\sim 5 \mathrm{~km} \mathrm{~s}^{-1}$ and a $K_{\mathrm{p}}$ of $\sim 190 \mathrm{~km} \mathrm{~s}^{-1}$. We find the Fe I signal at $K_{\mathrm{p}}=200 \pm 41.1 \mathrm{~km} \mathrm{~s}^{-1}, v_{\text {sys }}=-7.3 \pm 5.8$ $\mathrm{km} \mathrm{s}^{-1}$ (see Fig. 3). The negative $v_{\text {sys }}$ offset is also consistent with those observed by Hoeijmakers et al. (2020b), Ben-Yami et al. (2020), Bourrier et al. (2020a), and Cabot et al. (2020) and is thus thought to be reliable. We thus judge any signal that lies within $3 \mathrm{~km} \mathrm{~s}^{-1}$ in $v_{\text {sys }}$ and $20 \mathrm{~km} \mathrm{~s}^{-1}$ of $K_{\mathrm{p}}$ of the Fe I signal presented in Gibson et al. (2020) to lie at the 'expected' position.

(iii) Whether the signal is detected in our injection tests. If a species is found as both a standard detection in the data and in injection, this increases confidence in the detection. Conversely, if no signal is found in injection for a species that presents a detected signal, we might assume that we do not in fact have the sensitivity to detect the species in question. Also, as we inject the signals at a velocity far from the prospective exoplanetary signal, this criterion accounts for cases where a model with very few lines 'aliases' similar lines found in other extant species, potentially leading to a spurious detection (see Co I, Section 4.3.8).

(iv) The presence of structure in the maps. This was assessed by a visual inspection to determine whether any peaks of similar size to the signal existed within the cross-correlation map.

(v) Whether the species has been detected before, whether in other analyses of WASP-121b (e.g. Ben-Yami et al. 2020; Cabot et al. 2020; Hoeijmakers et al. 2020b), or in other UHJs such as KELT-9b (Hoeijmakers et al. 2018, 2019) or KELT-20b (e.g. Casasayas-Barris et al. 2019; Nugroho et al. 2020a). Though we elect to remain as model agnostic as possible, and thus assign no detectability criteria 
Table 2. Parameters for detected signals presented in this work, in order of overall confidence. Here $\sigma, K_{\mathrm{p}}$, and $v_{\text {sys }}$ are the signal detection significance and velocity location from the combined red+blue maps (apart from Ti I, for which the blue arm results are presented). Uncertainties for these quantities were estimated from the FWHM of the signal, converted to a standard deviation. $T$ and $P_{\text {cloud }}$ are the temperature and cloud deck pressure level parameters of the model for which the strongest signal was retrieved for the species in question (shown in Fig. 7). The SYSREM iterations column provides the number of iterations that resulted in the strongest signal for the species.

\begin{tabular}{|c|c|c|c|c|c|c|}
\hline Species & $\sigma$ & $\mathrm{K}_{p}\left(k m s^{-1}\right)$ & $v_{\text {sys }}\left(k m s^{-1}\right)$ & $\mathrm{T}(K)$ & $\mathrm{P}_{\text {cloud }}($ bar) & SYSREM iterations \\
\hline $\mathrm{Fe}_{I}$ & 8.8 & $200 \pm 41.1$ & $-7.3 \pm 5.8$ & 3000 & 0.05 & 10 \\
\hline CaII & 6.4 & $235 \pm 54.4$ & $-7.3 \pm 11$ & 4500 & 1 & 10 \\
\hline $\mathrm{Cr} I$ & 5.0 & $198 \pm 75.6$ & $-5.5 \pm 4.6$ & 2000 & 1 & 10 \\
\hline$V_{I}$ & 4.4 & $226 \pm 44.2$ & $-5.5 \pm 7.0$ & 2000 & $5 \times 10^{-5}$ & 10 \\
\hline $\mathrm{CaI}$ & 4.2 & $199 \pm 24.6$ & $-5.5 \pm 3.5$ & 3000 & $5 \times 10^{-5}$ & 10 \\
\hline$H_{I}$ & 5.7 & $236 \pm 82.4$ & $-4.6 \pm 12$ & 7000 & 1 & 15 \\
\hline$K_{I}$ & 4.4 & $198 \pm 21.2$ & $-6.4 \pm 2.7$ & 3000 & $5 \times 10^{-5}$ & 20 \\
\hline Sc II & 4.2 & $212 \pm 26.3$ & $5.5 \pm 5.8$ & 2000 & $5 \times 10^{-5}$ & 20 \\
\hline$M n_{I}$ & 4.0 & $217 \pm 28.0$ & $-4.6 \pm 3.9$ & 2000 & 0.05 & 10 \\
\hline Co II & 3.8 & $218 \pm 15.7$ & $-7.3 \pm 1.9$ & 3000 & 1 & 15 \\
\hline $\mathrm{NiI}$ & 3.7 & $221 \pm 9.3$ & $-11 \pm 1.6$ & 2500 & 0.05 & 20 \\
\hline CoI & 3.6 & $198 \pm 29.3$ & $-13 \pm 3.5$ & 2000 & 0.05 & 10 \\
\hline $\mathrm{Cu} I$ & 4.6 & $203 \pm 11.0$ & $-11 \pm 2.3$ & 3000 & $5 \times 10^{-5}$ & 10 \\
\hline$S r I$ & 4.0 & $203 \pm 18.7$ & $0.9 \pm 1.9$ & 2500 & 0.001 & 20 \\
\hline$V_{I I}$ & 3.7 & $210 \pm 8.1$ & $-11 \pm 1.6$ & 2000 & 1 & 20 \\
\hline$T i I^{a}$ & 3.6 & $234 \pm 27.2$ & $-5.1 \pm 1.7$ & 2000 & $5 \times 10^{-5}$ & 10 \\
\hline$S r I I$ & 3.6 & $201 \pm 18.7$ & $-0.9 \pm 8.5$ & 5000 & $5 \times 10^{-5}$ & 10 \\
\hline Ti II & 3.4 & $204 \pm 17.0$ & $-14 \pm 3.5$ & 7000 & 0.05 & 10 \\
\hline $\mathrm{Fe}$ II & 3.2 & $217 \pm 9.8$ & $-9.2 \pm 1.9$ & 3000 & 1 & 10 \\
\hline$M g I$ & 2.9 & $198 \pm 14.0$ & $-7.3 \pm 2.7$ & 3000 & 1 & 15 \\
\hline
\end{tabular}

Note. ${ }^{a}$ Blue arm only.

based upon whether a species is expected in WASP-121b via models, the actual previous detection of a species in WASP-121b or indeed in other UHJs lends credibility to a previous detection.

\subsection{Results of cross-correlation and injection tests}

A list of the 20 signals of interest and their parameters, sorted by the criteria fulfilled, is presented in Table 2. The detection criteria fulfilled for each species are elaborated in Table 3.

The cross-correlation maps and slices through the maps at the relevant $K_{\mathrm{p}}$, with comparison to the injection tests, are found in Figs 3-6. In each case, we have chosen the result from model with the temperature and $P_{\text {cloud }}$ that provides the largest detection significance: these models can be found in Fig. 7. Similarly, we present the results from the number of SYSREM iterations that maximize the signal strength: however, we note that all signals are retrievable at 10 iterations. We reiterate here that the temperature and $P_{\text {cloud }}$ parameters are largely degenerate with other parameters; in each case, the 'best' model is simply the one that provides the closest match to the exoplanetary signal out of those tested in terms of line strength above the continuum. The detection significances presented are those found using the more simplistic method of dividing the map by the standard deviation outside of the signal area. Phase shuffling (as described in Section 3.3) was found to vary the detection significance very slightly but otherwise produced very similar results, so we have reverted to the simpler, less computationally intensive method here.

Only the eight signals found to fulfil four or five of our detection criteria are classed in this work as detections. The strongest and most reliable signals are of $\mathrm{Fe} \mathrm{I,} \mathrm{Cr} \mathrm{I,} \mathrm{V} \mathrm{I,} \mathrm{Ca} \mathrm{I,} \mathrm{and} \mathrm{Ca} \mathrm{II,} \mathrm{fulfilling} \mathrm{five} \mathrm{out}$ of five of the detection criteria; we classify these as strong detections. Relatively interesting signals were also found of $\mathrm{HI}, \mathrm{KI}$, and Sc II, fulfilling four criteria each, and are classified in this work as weak detections.

Tentative signals were also found of Mn I, Co II, Ni I, and Co I: these signals were less reliable, fulfilling three of the detection criteria. Weaker hints were found of CuI, SrI, V II, Ti I, and Sr II. These satisfied only 1-2 of our detection criteria. We do not claim any of these weaker signals as possible detections, and present them only to provide interesting avenues of exploration for future work or as some small additional evidence for already-discovered species. Finally, three species show signals below the detection threshold of $3.5 \sigma: \mathrm{Fe}$ II, Mg I, and Ti II. We class these as technically insignificant but worthy of further discussion. Further discussion of each of these signals in depth is presented in the following section.

\subsection{Individual species results}

Given the large number of interesting signals, in the following section we discuss the eight signals classed as either strong or weak detections individually, with reference to the cross-correlation maps shown in Figs 3-5. The weak signals are discussed as a whole in Section 4.3.8 and are shown in Figs 5 and 6.

\subsection{1 $\mathrm{FeI}$}

As expected from previous work, we detect the Fe I signal strongly at $8.8 \sigma$, within range of the expected location $\left(K_{\mathrm{p}}=200 \pm 41.1\right.$ $\mathrm{km} \mathrm{s}^{-1}, v_{\text {sys }}=-7.3 \pm 5.8 \mathrm{~km} \mathrm{~s}^{-1}$ ), in the combined blue+red map. This independent confirmation of our previous detection using both arms of the data fulfils all five of our detection criteria and serves as a benchmark test for our analysis, as the detection was previously well characterized by Gibson et al. (2020) using the blue arm of this data set and confirmed by Cabot et al. (2020), Bourrier et al. 
Table 3. Detected signals and the detection criteria they fulfil from Section 4.1. References for WASP-121b: [1] Gibson et al. (2020); [2] Ben-Yami et al. (2020); [3] Hoeijmakers et al. (2020b); [4] Cabot et al. (2020); [5] Bourrier et al. (2020a); [6] Borsa et al. (2021); and [7] Sing et al. (2019). References for other hot Jupiters: [A] Nugroho et al. (2020b); [B] Ehrenreich et al. (2020); [C] Stangret et al. (2020); [D] Hoeijmakers et al. (2018); [E] Nugroho et al. (2020a); [F] Casasayas-Barris et al. (2019); [G] Turner et al. (2020); [H] Hoeijmakers et al. (2019); [I] Astudillo-Defru \& Rojo (2013); [J] Sing et al. (2011); [K] Colón et al. (2012); [L] Sedaghati et al. (2016); and [M] Vidal-Madjar et al. (2013). Citations for species found in other hot Jupiters are non-exhaustive.

\begin{tabular}{|c|c|c|c|c|c|c|c|}
\hline Species & $\begin{array}{l}\text { Significance } \\
\quad>3.5 \sigma\end{array}$ & $\begin{array}{l}\text { Expected } \\
\text { position }\end{array}$ & $\begin{array}{l}\text { Present in } \\
\text { injection }\end{array}$ & $\begin{array}{l}\text { Minimal noise in } \\
\text { map }\end{array}$ & $\begin{array}{l}\text { Detected in } \\
\text { WASP-121b? }\end{array}$ & $\begin{array}{l}\text { Detected in } \\
\text { other HJ? }\end{array}$ & $\begin{array}{l}\text { Strength of } \\
\text { signal }\end{array}$ \\
\hline $\mathrm{Fe} \mathrm{I}$ & $\checkmark$ & $\checkmark$ & $\checkmark$ & $\checkmark$ & {$[1,2,3,4,5,6]$} & {$[\mathrm{A}, \mathrm{B}, \mathrm{C}, \mathrm{D}]$} & Strong \\
\hline Ca II & $\checkmark$ & $\checkmark$ & $\checkmark$ & $\checkmark$ & {$[6]$} & {$[\mathrm{E}, \mathrm{F}, \mathrm{G}]$} & Strong \\
\hline $\mathrm{CrI}$ & $\checkmark$ & $\checkmark$ & $\checkmark$ & $\checkmark$ & {$[2,3,6]$} & {$[\mathrm{H}]$} & Strong \\
\hline V I & $\checkmark$ & $\checkmark$ & $\checkmark$ & $\checkmark$ & {$[2,3,6]$} & - & Strong \\
\hline $\mathrm{CaI}$ & $\checkmark$ & $\checkmark$ & $\checkmark$ & $\checkmark$ & [3] & {$[\mathrm{I}]$} & Strong \\
\hline HI & $\checkmark$ & $\checkmark$ & $x$ & $\checkmark$ & {$[4,6]$} & {$[\mathrm{F}, \mathrm{G}]$} & Tentative \\
\hline $\mathrm{K}_{\mathrm{I}}$ & $\checkmark$ & $\checkmark$ & $\times$ & $\checkmark$ & [6] & {$[\mathrm{J}, \mathrm{K}, \mathrm{L}]$} & Tentative \\
\hline Sc II & $\checkmark$ & $x$ & $\checkmark$ & $\checkmark$ & - & {$[\mathrm{H}]$} & Tentative \\
\hline Mn I & $\checkmark$ & $\checkmark$ & $\checkmark$ & $x$ & - & - & Weak \\
\hline Co II & $\checkmark$ & $\checkmark$ & $\checkmark$ & $x$ & - & - & Weak \\
\hline Ni I & $\checkmark$ & $x$ & $\checkmark$ & $x$ & [3] & - & Weak \\
\hline Co I & $\checkmark$ & $x$ & $\checkmark$ & $x$ & - & {$[\mathrm{H}]$} & Weak \\
\hline $\mathrm{Cu} \mathrm{I}$ & $\checkmark$ & $x$ & $\times$ & $\checkmark$ & - & - & Very weak \\
\hline SrI & $\checkmark$ & $\times$ & $\checkmark$ & $x$ & - & - & Very weak \\
\hline V II & $\checkmark$ & $x$ & $\checkmark$ & $x$ & - & - & Very weak \\
\hline Ti I & $x^{a}$ & $\checkmark$ & $\checkmark$ & $x$ & - & - & Very weak \\
\hline Sr II & $\checkmark$ & $x$ & $\times$ & $x$ & - & {$[\mathrm{H}]$} & Very weak \\
\hline Ti II & $x$ & $x$ & $\checkmark$ & $x$ & - & {$[\mathrm{D}, \mathrm{H}]$} & Insignificant \\
\hline Fe II & $x$ & $\times$ & $\checkmark$ & $\times$ & {$[2,6,7]$} & {$[\mathrm{C}, \mathrm{D}, \mathrm{F}, \mathrm{H}]$} & Insignificant \\
\hline Mg I & $x$ & $\checkmark$ & $x$ & $x$ & {$[3,6]$} & {$[\mathrm{H}, \mathrm{M}]$} & Insignificant \\
\hline
\end{tabular}

Note. ${ }^{a}$ Significance $>3.5$ in blue arm only.

(2020a), Ben-Yami et al. (2020), and Hoeijmakers et al. (2020b) in the HARPS data set. Our detection is consistent with these works. Although we detect the Fe I signal at a larger negative $v_{\text {sys }}$ offset than the values reported in the aforementioned works, we note that UVES is less stable in wavelength than HARPS, and less sensitive, with an average resolution element of $\sim 3.15 \mathrm{~km} \mathrm{~s}^{-1}$. In addition, this analysis was performed with an independent reduction and analysis pipeline.

\subsubsection{CaII}

We retrieve a strong $(\sim 6.3 \sigma)$ signal for $\mathrm{Ca}$ II in the expected location $\left(K_{\mathrm{p}}=236 \pm 54.4 \mathrm{~km} \mathrm{~s}^{-1}, v_{\text {sys }}=-7.3 \pm 11 \mathrm{~km} \mathrm{~s}^{-1}\right)$. As shown in Fig. 4, we do not detect a significant signal in the blue arm of our data, which contains the strong $\mathrm{Ca} I \mathrm{II} \mathrm{H} \& \mathrm{~K}$ lines. We also did not detect a signal in the blue arm for any of our original Ca II injection tests. We theorize that this is due to the presence of extremely broad H\& K lines in the stellar spectrum. Our data will have low $\mathrm{S} / \mathrm{N}$ in the vicinity of these broad stellar lines, especially after optimal weighting. A similar effect was observed by Seidel et al. (2020) for sodium in the high-resolution transmission spectrum of the bloated super-Neptune WASP-166b. The majority of our detected signal comes instead from the strong triplet at the redward end of our red arm data.

Though Ca II was not found in our original injection tests using models limited by the assumption of hydrostatic equilibrium, it is recovered easily when we scale the injected models to account for a higher temperature and escaping atmosphere, as described in Section 3.4. We also recover stronger signals at higher temperatures, with our strongest signal found using a model generated at $4500 \mathrm{~K}$. We thus surmise, with reference to Sing et al. (2019) and Borsa et al. (2021), that Ca II exists mainly in the hot extended exosphere of WASP-121b. This is discussed further in Section 5. Ca II has previously been detected in the atmosphere of the UHJ KELT20b/MASCARA-2b by Casasayas-Barris et al. (2019) and Nugroho et al. (2020a), where it is also theorized to be part of an escaping atmosphere.

\subsubsection{CrI and VI}

We confirm the detection of Cr I by Ben-Yami et al. (2020), Hoeijmakers et al. (2020b), and Borsa et al. (2021), recovering the signal at $5.0 \sigma$ at a position consistent with these works $\left(K_{\mathrm{p}}=198+76\right.$ $\left.\mathrm{km} \mathrm{s}^{-1}, v_{\text {sys }}=-5.5 \pm 4.6 \mathrm{~km} \mathrm{~s}^{-1}\right)$ and fulfilling all five of our detection criteria. As can be seen in Fig. 1, the signals are found in slightly different velocity locations in the blue and red arms. We discuss the variation in offsets in $v_{\text {sys }}$ further in Section 5 .

We also confirm the detections of VI (Ben-Yami et al. 2020; Hoeijmakers et al. 2020b; Borsa et al. 2021), recovering the signal at $4.4 \sigma$ within range of the expected location $\left(K_{\mathrm{p}}=226 \pm 44.2\right.$ $\mathrm{km} \mathrm{s}^{-1}, v_{\text {sys }}=-5.5 \pm 7.0 \mathrm{~km} \mathrm{~s}^{-1}$ ) and fulfilling all five of our detection criteria.

\subsection{4 $\mathrm{CaI}$}

We retrieve a Ca I signal of $4.2 \sigma$ close to the expected position, at $K_{\mathrm{p}}=199 \pm 24.6 \mathrm{~km} \mathrm{~s}^{-1}$ and $v_{\text {sys }}=-5.5 \pm 3.5 \mathrm{~km} \mathrm{~s}^{-1}$. There is a small amount of structure in the cross-correlation map that, upon inspection of the maps from the individual arms, seems to result from an imperfect removal of the stellar Ca I signal in the red arm. This imperfect removal may be due to the fact that our red arm data are detrended using SYSREM in the rest frame of the Earth, in order to optimize the removal of the telluric spectrum, which is usually far more dominant within this wavelength range. Because of this structure in the cross-correlation map, this detection fulfils 

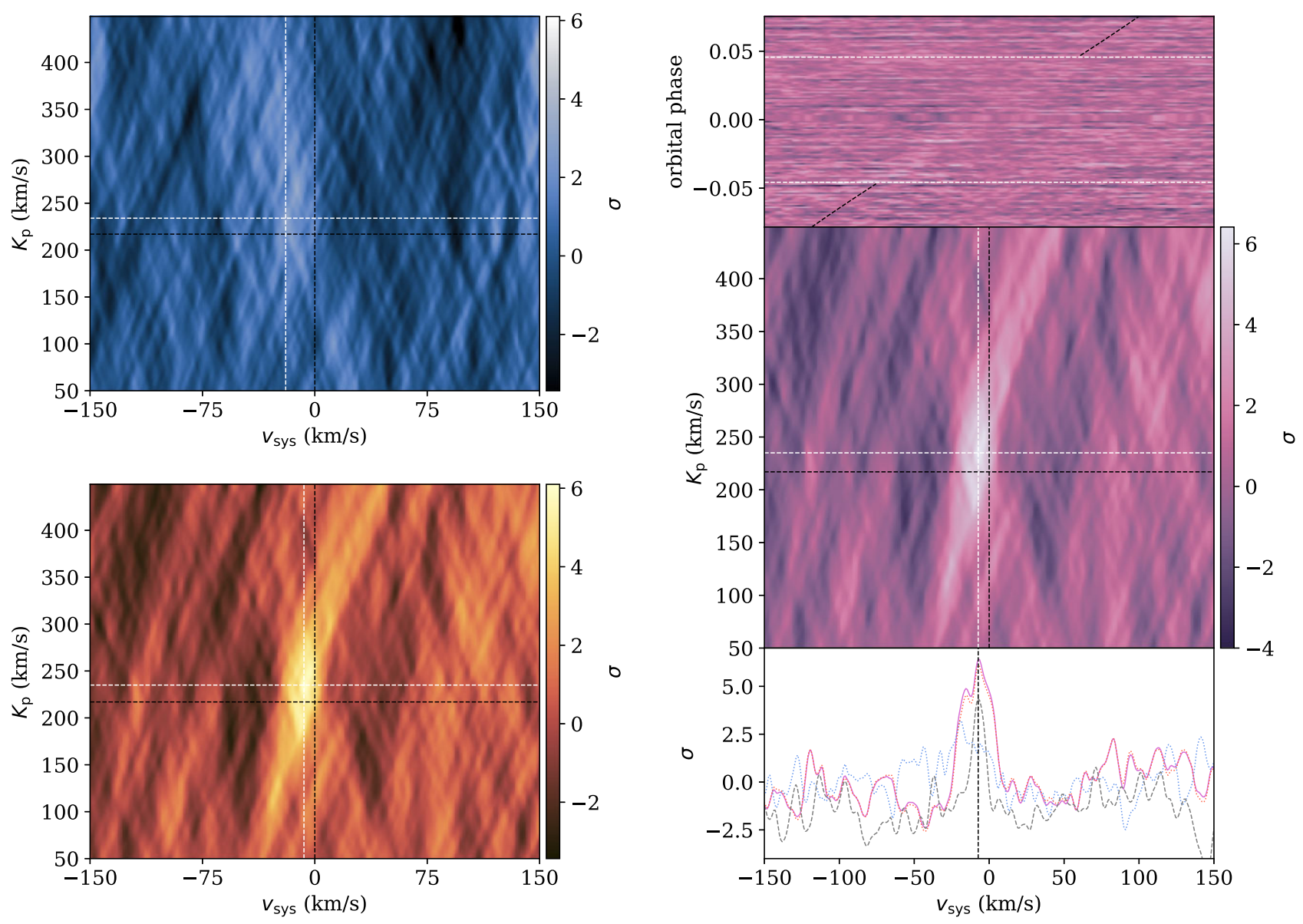

Figure 4. Results from cross-correlation for Ca II. Left: cross-correlation velocity maps for the blue arm (top) and the red arm (bottom), showing lack of signal in the blue arm. Right: as results presented in Fig. 3. The red and blue dotted lines in the bottom right-hand plot represent slices through the red and blue maps at the detected $K_{\mathrm{p}}$. The grey dashed line represents the injected signal for the same model with the line strength boosted to $\sim 0.25-0.3 R_{\mathrm{p}} / R_{\star}$ and adjusted to have the same $v_{\text {sys }}$ offset as the detected signal.

only four of our five detection criteria. However, Ca I was detected in the HARPS data sets by Hoeijmakers et al. (2020b), giving further confidence in its detection in the UVES data set.

\subsection{5 $\mathrm{HI}_{\mathrm{I}}$}

We retrieve a strong but exceptionally diffuse signal for $\mathrm{H} \mathrm{I}$ at $5.7 \sigma$, at the expected location of $K_{\mathrm{p}}=236 \pm 82.4 \mathrm{~km} \mathrm{~s}^{-1}$ and $v_{\text {sys }}=$ $-4.6 \pm 12 \mathrm{~km} \mathrm{~s}^{-1}$. The diffuse nature of the signal can be attributed to the broad nature of the Balmer lines in both our model and in the signal present within the data. The high temperature at which we detect our strongest signal $(7000 \mathrm{~K})$ would support this hypothesis, as $\mathrm{HI}$ is presumably, like Ca II, present in the extended atmosphere. This broadening may also explain why the $\mathrm{H}$ I signal is not present in our injection tests, even when the $\mathrm{H} \alpha$ line is scaled upwards to match the height of the feature found by Cabot et al. (2020) in WASP-121b using differential transit analysis.

\subsection{6 $K_{I}$}

The KI signal is recovered at $4.4 \sigma$ and is found in the expected position, at $K_{\mathrm{p}}=198 \pm 21.2 \mathrm{~km} \mathrm{~s}^{-1}$ and $v_{\text {sys }}=-6.42 .7 \mathrm{~km} \mathrm{~s}^{-1}$; however, it is only present in injection tests at higher values of $P_{\text {cloud }}$ than the model we found to produce the strongest signal. Potassium is thought to be a commonly detectable component of hot Jupiter atmospheres (Fortney et al. 2010, see also detections by e.g. Sing et al. 2011; Colón et al. 2012; Sedaghati et al. 2016), but it is expected to be largely ionized at the temperatures probed at the limb of WASP121b. However, $\mathrm{K}$ was also detected in the analysis of ESPRESSO data of WASP-121b presented by Borsa et al. (2021): our detection in the UVES data thus adds additional confidence to its presence. It is possible that differential transit analysis focused on strong $\mathrm{K}$ lines could further confirm this detection (see Gibson et al. 2019, for an example with UVES). This method could additionally explore other species with few, strong lines in the wavelength region; previous detections made with this method in WASP-121b include Cabot et al. (2020)'s detection of both the Na I doublet and an extended $\mathrm{H} \alpha$ feature attributed to atmospheric escape.

\subsubsection{SC II}

We retrieve a Sc II signal at $4.2 \sigma$. The signal seems to be fairly clear and well defined within the cross-correlation maps, with a small amount of structure, and at $212 \pm 26.3 \mathrm{~km} \mathrm{~s}^{-1}$ is well placed in $K_{\mathrm{p}}$, but the position of the signal in systemic velocity is extremely unusual: the offset in $v_{\text {sys }}$ is positive, at $5.5 \pm 5.8 \mathrm{~km} \mathrm{~s}^{-1}$. Sc II has so far been discovered only in KELT-9b (Hoeijmakers et al. 2019), suggesting that if this signal is physical, we are probing a high-temperature 

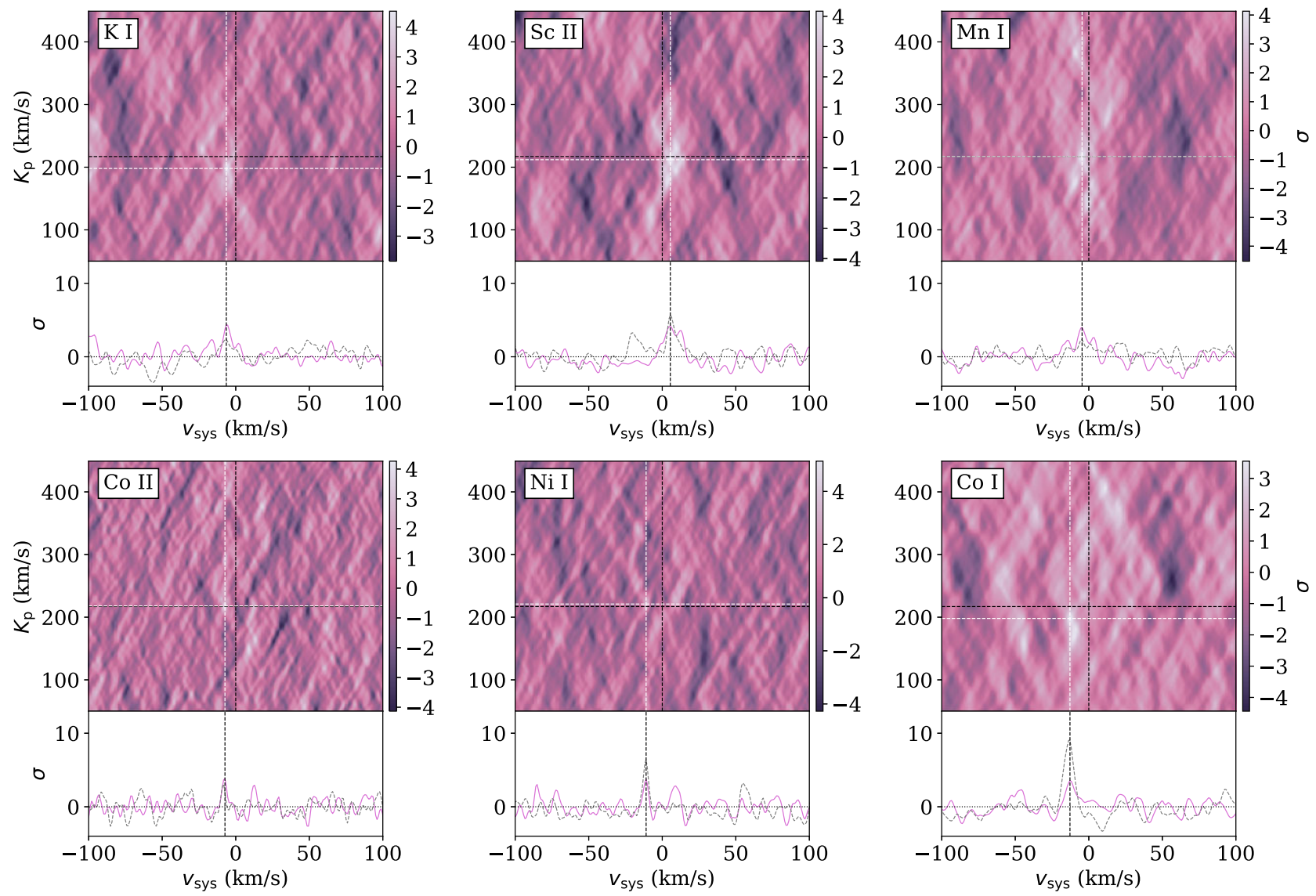

Figure 5. As Fig. 3, but for KI, Sc II, Mn I, Co II, Ni I, and Co I. Note that the summed cross-correlation functions (CCFs) have been omitted from this figure due to the lack of visible trace.
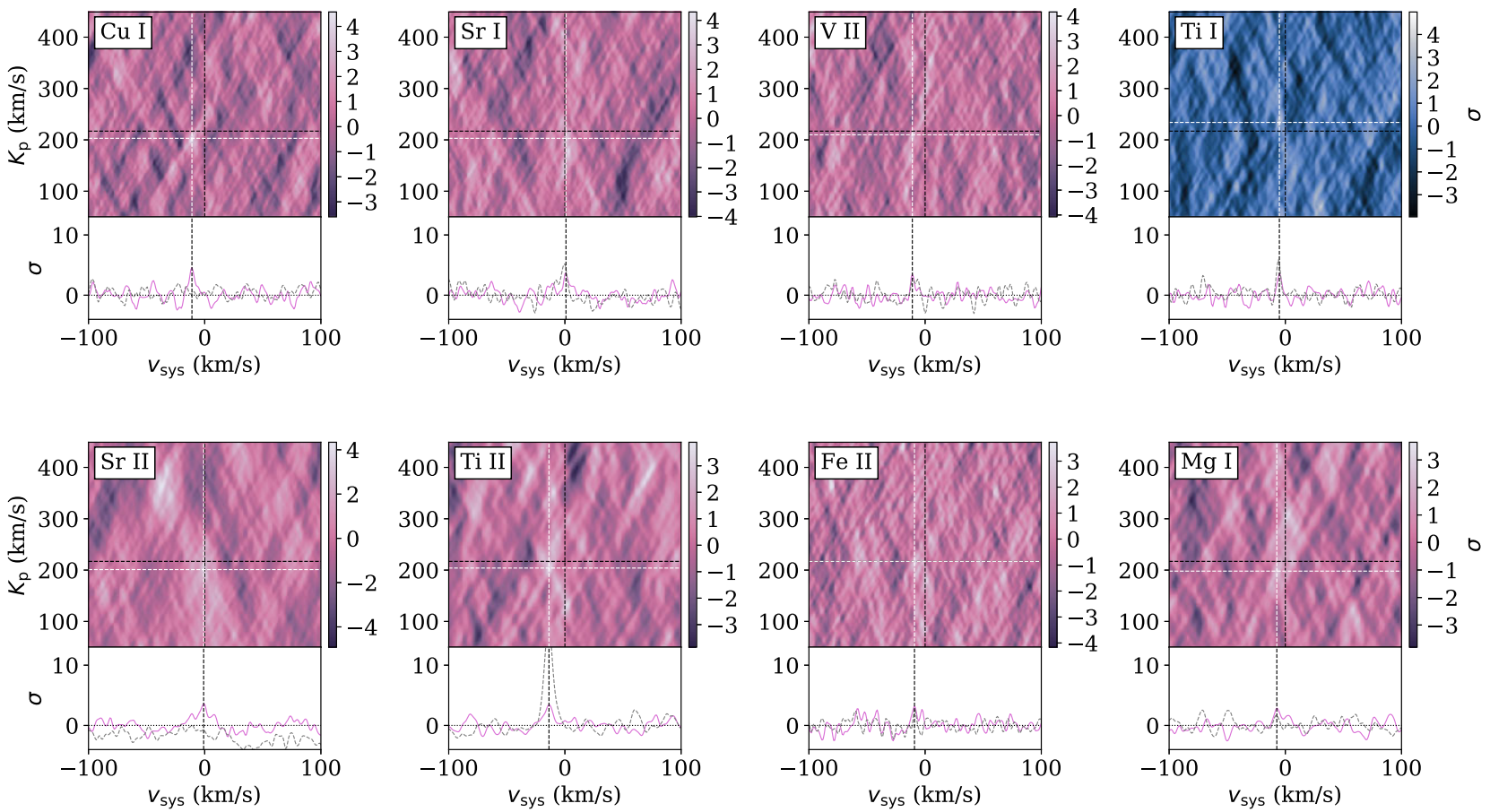

Figure 6. As Fig. 5, but for Cu I, Sr I, V II, Ti I, Sr II, Ti II, Fe II, and V II. Note that for Ti I, the velocity map presented is from the blue arm only, as the signal did not appear in the combined blue+red velocity map. 


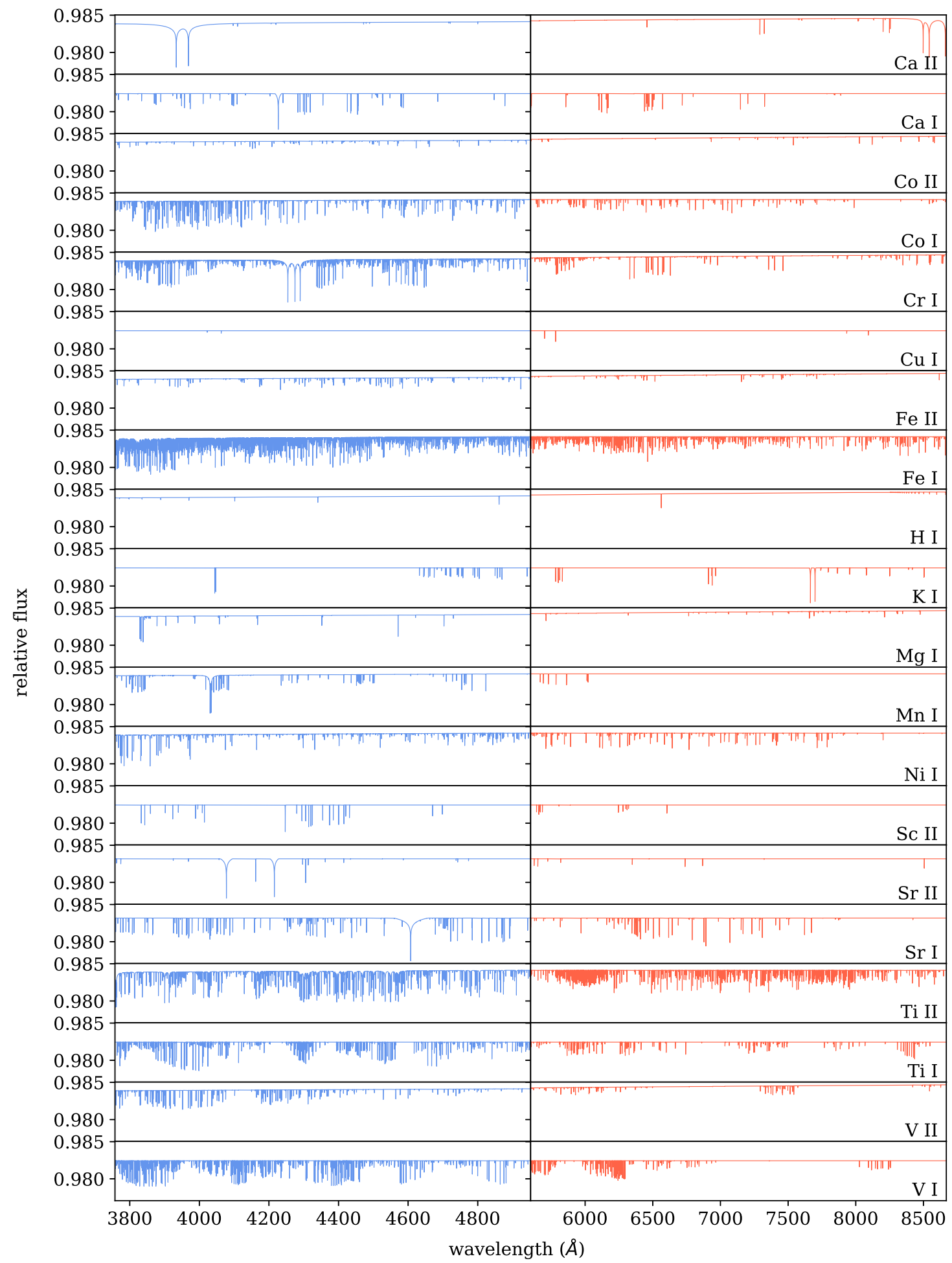

Figure 7. The model transmission spectra used to recover the 20 signals presented in this work. The left-hand column shows the wavelength range of the blue arm of our data. The right-hand column shows the range of the red arm. Parameters of these models can be found in Table 2. All ion models are shown before the scaling described in Section 3.4.

region, perhaps in the exosphere (similarly to Ca II, Section 4.3.2). This could also partially explain the very different $v_{\text {sys }}$. However, unlike Ca II, we do not retrieve the signal at a higher significance using higher temperature models. Because of this, and due to the unusual location of the signal, we encourage further investigation.

\subsubsection{Other signals}

While we classify none of the following as detections, we also find weak signals for Mn I, Co II, Ni I, and CoI; very weak signals for of CuI, SrI, V II, TiI, and SrII; and technically insignificant but 

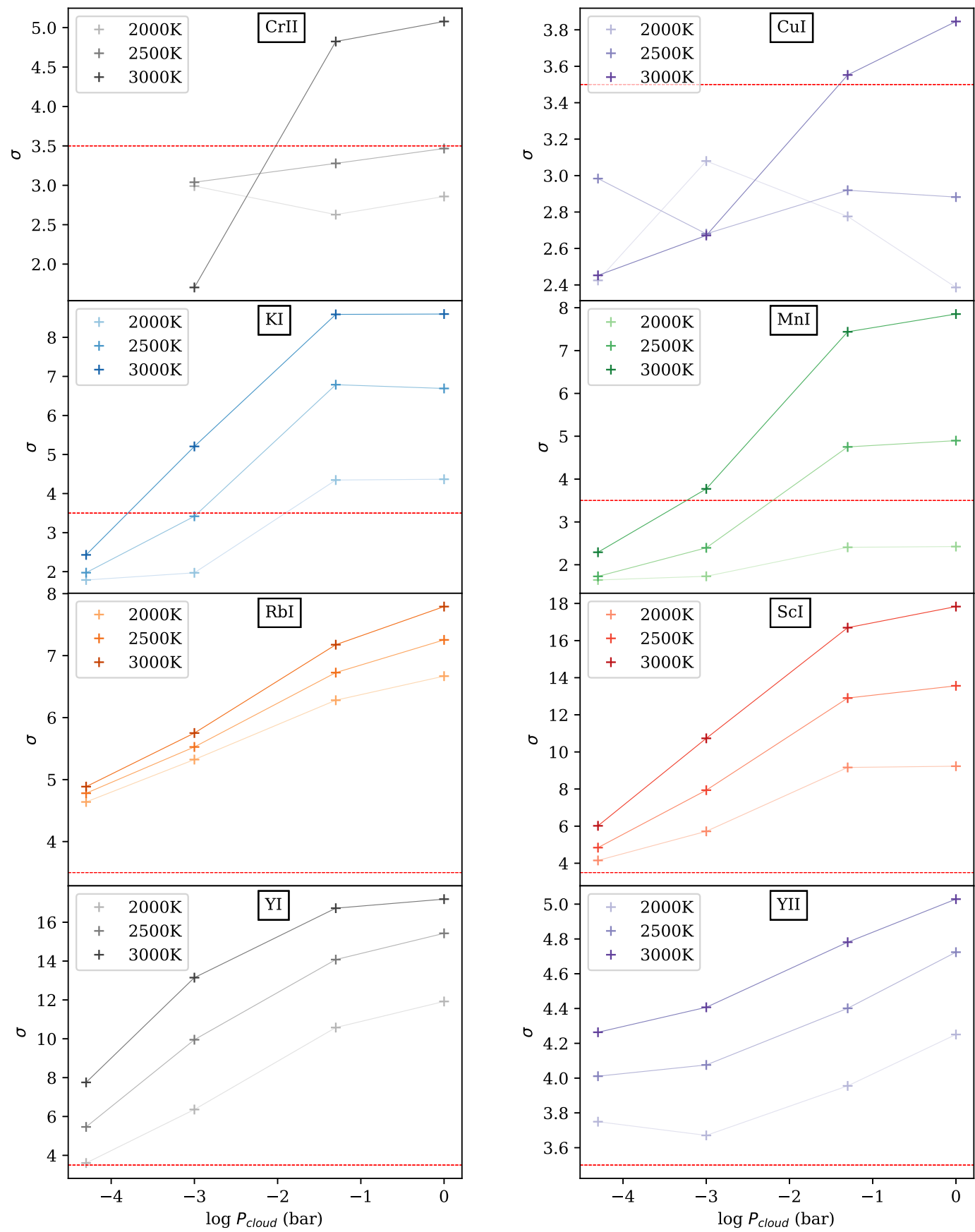

Figure 8. Results from injection tests for notable species, showing $\sigma$ versus $P_{\text {cloud }}$ for every model injected. The red dotted line indicates $\sigma=3.5$, in accordance with the detection criteria in Section 4.1.

interesting signals of Fe II, Mg I, and Ti II. Further details of these weaker signals can be found in Table 2 and Figs 5 and 6 .

We find a signal for MnI at $4.0 \sigma$ and at the expected location. Though the signal appears quite clear, there is, however, a lot of structure in the map: the signal seems to have a 'trail' pointing downwards in $K_{\mathrm{p}}$, and there is an additional peak found at $K_{\mathrm{p}}=\sim 375 \mathrm{~km} \mathrm{~s}^{-1}$. Additionally, the model for which we present the highest detection significance for Mn I does not result in a detectable signal when injected, although Mn I was found to be detectable in injection at higher temperatures/lower values for $P_{\text {cloud }}$ (see Fig. 8). For these reasons, we do not claim it as a detection. To date, Mn I has not been confirmed in an exoplanet atmosphere. However, it was theorized by Lothringer et al. (2020) to be one of many species contributing to the increased transit depth at short wavelengths seen in low-resolution transmission spectra of UHJs, including WASP-121b (Evans et al. 2018).

A faint Co II signal is detected at $3.8 \sigma$, close to the expected location. A Co II signal is also seen faintly in injection. However, the presence of structure in the map at a similar significance level greatly reduces our confidence in this signal, and Co II has not yet been detected in an exoplanet atmosphere.

$\mathrm{Mg}$ I and Ni I have been previously detected by Hoeijmakers et al. (2020b) in WASP-121b, with Mg I also being detected by Borsa et al. (2021). We do not recover either signal strongly. The Ni I signal is detected at $3.7 \sigma$, with a large negative $v_{\text {sys }}$ offset and much structure 
in the CCF map. We detect only a very weak potential Mg I signal, at $2.9 \sigma$. Our comparatively weaker signals for these species may be due to the higher resolution of HARPS and ESPRESSO at blue wavelengths and the wavelength coverage gap in our data between the red and blue arms. This gap, which is covered by the HARPS and ESPRESSO transits explored by Hoeijmakers et al. (2020b) and Borsa et al. (2021), contains a strong Mg I triplet at $\sim 5200 \AA$. We surmise that our lack of coverage of this triplet is responsible for the faintness of our $\mathrm{Mg}$ I signal. Though other strong $\mathrm{Mg}$ I lines exist at the very blueward end of the blue arm of our data, this region of our spectra is low in $\mathrm{S} / \mathrm{N}$ and is downweighted heavily in our analysis.

We retrieve a faint Co I signal at 3.6 $\sigma$. However, while its location in $K_{\mathrm{p}}$ is within the expected range, the signal position is at a much greater negative offset in $v_{\text {sys }}$, at $-12.3 \pm 3.5 \mathrm{~km} \mathrm{~s}^{-1}$. A similarly large negative offset is found in several other potential signals, such as Ni I, and is discussed further in Section 5. The presence of similarly sized structure in the map also reduces our confidence in this signal. Evidence of Co I has previously been found in KELT-9b by Hoeijmakers et al. (2019); Hoeijmakers et al. (2020b) searched for Co I in WASP-121b, but did not detect a signal.

The signal we recover for $\mathrm{Cu} \mathrm{I}$ is surprisingly high in significance $(4.6 \sigma)$, and presents a cross-correlation map largely free of similarly scaled structure. While the $v_{\text {sys }}$ offset, at $-11 \pm 2.3 \mathrm{~km} \mathrm{~s}^{-1}$, is anomalous, it is no more so than several other signals retrieved by our methodology. However, examination of the model (found in Fig. 7) shows that there are few lines present in the model over the wavelength range of our data. The signal appears to originate from just four lines in the red arm, none of which are particularly strong. Additionally, this species is not well retrieved in our injection tests, especially not at the temperature and cloud-deck level used to recover the original signal. It is possible that these four lines are 'aliasing' lines found in other extant species, producing a false positive. If this signal is spurious, as we suspect, then it justifies our use of a more elaborate set of detection criteria than simply the detection significance in $\sigma$ to judge the reliability of our detections.

Both Sr I and Sr II show faint signals, at $4.0 \sigma$ and $3.6 \sigma$, respectively. Sr II was previously tenuously detected by Hoeijmakers et al. (2019) in KELT-9b; similarly to their work, we also find a lot of positive structure in our cross-correlation map, casting doubt upon the veracity of the signals. We also recover a V II signal at $3.7 \sigma$, which if physical would indicate that enough of the V I is being ionized to be detectable in transmission.

We also find a faint $3.2 \sigma$ signal for Fe II, but at a large negative $v_{\text {sys }}$ offset. Sing et al. (2019) found strong evidence of escaping Fe II in their near-ultraviolet (NUV) observations of WASP-121b, and an Fe II signal was recovered by both Ben-Yami et al. (2020) and Borsa et al. (2021). However, previous work on the blue arm of the data set presented here by Gibson et al. (2020) did not retrieve a Fe II signal, and Hoeijmakers et al. (2020b) could not repeat Ben-Yami et al. (2020)'s detection in the HARPS spectra. It is possible that variations in methodology are responsible for the elusive nature of this signal, or perhaps even some intrinsic variation in the signal itself caused by atmospheric dynamics. Alternatively, it is also possible that the putative Fe II signal found in cross-correlation here is simply an unfortunately located noise peak, and our analysis is not sensitive to this species. It was suggested by Gibson et al. (2020) that the Fe II lines may be significantly broadened due to the larger velocity range within the escaping exosphere, leading to their removal during pre-processing and a resulting non-detection at high resolution. Fe II is readily detected in our injection tests at $\sim 12 \sigma$ when the injected model is scaled so the features match those found in Sing et al. (2019) (see Section 3.4), suggesting that the low number of spectral lines in the model is not necessarily responsible for previous non-detections. A simple test in which we broadened the injected spectral lines with a Gaussian kernel did, however, reduce the strength of the recovered injected signal by as much as $\sim 5 \sigma$

Finally, we detect some small hints of both Ti I and Ti II. Ti I is seen only in the blue arm of the data at $3.6 \sigma$, close to the expected position. We attribute its lack of presence in the red+blue combined map to destructive addition with a negative noise peak seen in the red map. Ti II is seen in the combined red+blue map at $3.4 \sigma$. Both maps contain similarly scaled structure, especially Ti II, which shows several peaks in the vicinity. Ti I was searched for - but not found - in the HARPS data set by both Ben-Yami et al. (2020) and Hoeijmakers et al. (2020b). Borsa et al. (2021) searched for both Ti I and Ti II with no result. We discuss the implications of this further in Section 5.

\subsection{Injection tests and non-detections}

Of the 43 species we searched for in WASP-121b, we detect no sign of the remaining 23. Notably, our injection tests show that several species we did not detect in our broad species search are nevertheless theoretically detectable using our methodology for given values of $T$ and $P_{\text {cloud }}$ : Y I, Y II, Rb I, Sc I, and Cr II. Additionally, though the specific models used to retrieve the $\mathrm{KI}, \mathrm{Cu}$, and Mn I signals do not present a signal in injection tests, these species are none the less detectable at higher values of $T$ or lower values of $P_{\text {cloud }}$. We present the results from these injection tests in Fig. 8.

Unlike in Merritt et al. (2020), we do not choose to set detection limits upon these species based upon the results of the injection tests, as here we chose to vary the position of the grey cloud deck $P_{\text {cloud }}$ rather than VMR. As discussed in Merritt et al. (2020), even had we varied VMR, any detection limits we set on abundances would be extremely contingent on the correct positioning of the cloud deck due to the unbreakable degeneracies present using our simplified atmospheric model.

\section{DISCUSSION}

Our methodology resulted in five detections, three tentative detections, and weak signs of a further nine different atomic species in the atmosphere of WASP-121b using a single UVES transit, standard cross-correlation methodology and model transmission spectra generated using a simple analytical approximation. Though we emphasize that only eight of these signals were strong enough to be classified as potential detections, our success nevertheless shows the effectiveness of high-resolution broad species searches in UHJs. We provide an independent confirmation of many of the species detected in the HARPS data set explored by Ben-Yami et al. (2020) and Hoeijmakers et al. (2020b) and the ESPRESSO data set explored in Borsa et al. (2021). We also add a novel (if tentative) detection of Sc II.

The presence alone of various species provides information on atmospheric conditions at the limb of WASP-121b. The existence of transition metals such as $\mathrm{Fe}, \mathrm{V}$, and $\mathrm{Cr}$ in $\mathrm{UHJ}$ atmospheres was predicted by theoretical work using stellar models by Lothringer et al. $(2018,2020)$ and Lothringer \& Barman (2019), who posited that the strong optical opacity of these species would be sufficient to drive the thermal inversion observed in emission (Evans et al. 2017; Mikal-Evans et al. 2019). It has also been posited (e.g. by Lothringer et al. 2020) that a forest of transition metal lines could be responsible for the strong opacity seen at wavelengths $<3000 \AA$ in transmission spectra of WASP-121b (Evans et al. 2018), WASP$76 \mathrm{~b}$ (Fu et al. 2020), and WASP-12b (Fossati et al. 2010; Haswell 
et al. 2012; Sing et al. 2013). Because of the dense region of overlapping lines they create, these species are extremely difficult to isolate at low resolution, highlighting the importance of highresolution observations for confirming the presence of such species. Additionally, the presence of any atomic species indicates that it is not substantially condensed out at the limb of WASP-121b, indicating that substantial regions of the limb of WASP-121b probed by our analysis are $>2000 \mathrm{~K}$ : below this temperature, we expect atomic species to largely have condensed out of the atmosphere (Hoeijmakers et al. 2020b).

Our strong detection of Ca II, tentative detection of $\mathrm{H}$ I, and weaker signals for ions such as Sc II, Co II, Sc II, Sr II, Ti II, and V II indicate that our analysis is probing much higher temperatures than might be expected from previous studies of the limb of WASP-121b (e.g. Evans et al. 2018; Gibson et al. 2020). Signs of Sc II and Sr II have previously only been detected in the extremely hot Jupiter KELT9b (>4000 K; Hoeijmakers et al. 2018, 2019). We therefore infer that these detections arise from the existence of a hot escaping atmosphere, as detected by Sing et al. (2019). This hypothesis is strengthened by the diffuse nature of our recovered $\mathrm{Ca}$ II and $\mathrm{HI}$ signals, suggesting the presence of broadened spectral lines in the transmission spectrum, and by the fact that despite the strength of our detection, Ca II is only detected in injection tests when we 'boost' the size of the spectral features in our models to $\sim 0.25-0.3 R_{\mathrm{p}} / R_{\star}$, similar to those found by Sing et al. (2019) for Fe II and Mg II.

Our analysis also discovered hints of Ti I and Ti II, neither of which were found in the HARPS data set by Ben-Yami et al. (2020) and Hoeijmakers et al. (2020b). Though we emphasize that we have found only the slightest trace of a signal for both species, their potential presence is intriguing. As mentioned, WASP-121b is known to host a temperature inversion due to the detection of water features in emission (Evans et al. 2017; Mikal-Evans et al. 2019, 2020), but so far no work to date has found convincing evidence of TiO, one of the molecules thought to be responsible for driving temperature in versions in the hottest of hot Jupiters (Hubeny, Burrows \& Sudarsky 2003; Fortney et al. 2008). Indeed, an explicit search for TiO in the red arm of this data by Merritt et al. (2020) using a similar methodology to that presented here found nothing. It has been suggested that perhaps absorption by atomic metals, most notably Fe I, may be responsible for the atmospheric heating driving the temperature inversion (Lothringer et al. 2018; Gibson et al. 2020), and that the lack of $\mathrm{Ti}$ and $\mathrm{TiO}$ in the atmosphere is due to a 'cold-trap' mechanism: the lower condensation temperature of $\mathrm{TiO}$ is causing it to condense out and become trapped in condensate form in cooler areas of the atmosphere (Lodders 2002; Hubeny et al. 2003; Spiegel, Silverio \& Burrows 2009; Parmentier, Showman \& Lian 2013; Parmentier et al. 2016; Beatty et al. 2017). In this scenario, Hoeijmakers et al. (2020b) posit that Ti would also be depleted, as chemical equilibrium would respond to the depletion of $\mathrm{TiO}$ by driving more and more atomic $\mathrm{Ti}$ into its oxide phase, causing further condensation until all Ti-bearing species are condensed out of their gas phase. A detection of Ti I and Ti II would seem to contradict this explanation, and render the lack of TiO even more mysterious. However, we emphasize that our signals are weak, and hope for further exploration with larger data sets consisting of more transits, perhaps at higher resolution, in order to confirm or rule out the presence of neutral or ionized atomic titanium.

We see a large amount of variance in the position of our signals, in both $v_{\text {sys }}$ and $K_{\mathrm{p}}$. The variation in $K_{\mathrm{p}}$ is to some extent expected. In addition to the approximate $\pm 15 \mathrm{~km} \mathrm{~s}^{-1}$ uncertainty on the 'true' value of $K_{\mathrm{p}}$ (calculated from parameters presented in Delrez et al. 2016), only a small portion of the planetary orbit is sampled during a transit, resulting in a large spread in $K_{\mathrm{p}}$ (Brogi et al. 2018). Similar variation is seen in Hoeijmakers et al. (2020b) and Ben-Yami et al. (2020), with Ben-Yami et al. (2020) seeing variance of $\sim 50 \mathrm{~km} \mathrm{~s}^{-1}$ in $K_{\mathrm{p}}$.

The potential sources of variation in $v_{\text {sys }}$ are more complex. The slightly differing signal location between red and blue arms seen in Fig. 1 is a fairly common feature of the detected signals presented in this work. If physical, it may signify that we are probing different atmospheric regimes with the two different wavelength ranges. However, given the known instability of UVES in wavelength when compared with more stable instruments like HARPS, and the different methods used to correct the wavelength solution for the blue and red arms outlined in Section 2, we suspect that this interarm variation in $v_{\text {sys }}$ is due to differences in alignment and wavelength solution accuracy between the blue and red arms.

We also see large shifts in $v_{\text {sys }}$ in the combined red+blue maps for a large number of the signals we recover. As previously mentioned, $\mathrm{Fe}$ I has been consistently recovered with a blueshifted $v_{\text {sys }}$ offset of -3 to $-5 \mathrm{~km} \mathrm{~s}^{-1}$ (Ben-Yami et al. 2020; Bourrier et al. 2020a; Cabot et al. 2020; Gibson et al. 2020; Hoeijmakers et al. 2020b), which has been attributed to the presence of atmospheric dynamics: namely, strong day-to-nightside winds, predicted to be on the order of $\approx 5 \mathrm{~km} \mathrm{~s}^{-1}$ (e.g. Kataria et al. 2016). Our Fe I signal is found at $-7.3 \mathrm{~km} \mathrm{~s}^{-1}$, broadly in agreement with previous measurements, especially considering that UVES is less stable in wavelength than HARPS, and less sensitive, with an average resolution element of $\sim 3.15 \mathrm{~km} \mathrm{~s}^{-1}$. However, many of the more tentative signals shown in Figs 5 and 6 are found at more variant $v_{\text {sys }}$ offsets. For example, Co I, Ni I, Co II, and several of the weakest signals are found at larger negative $v_{\text {sys }}$ offsets, on the order of $\approx-10 \mathrm{~km} \mathrm{~s}^{-1}$. If physical, it could perhaps be possible that these species are predominantly found in areas of the atmosphere for which the wind speed is even higher, leading to a larger blueshift in the signal. However, winds this strong in the atmosphere are not predicted by current general circulation models (GCMs) of UHJs (Kataria et al. 2016). Instead, it is possible that a variant blueshift may be caused by material escaping the planet, pushed away from the planet and the star by stellar pressure. Hydrogen has been observed to reach velocities of up to $100 \mathrm{~km} \mathrm{~s}^{-1}$ in the escaping tail of HD 209458b (Vidal-Madjar et al. 2003; Ben-Jaffel 2007). Speeds in the upper atmosphere could vary between species as a result of either differing atomic weight or ionization state (due to interaction with magnetic fields).

Additionally, some species such as Sc II and Mg I show a redshift in $v_{\text {sys }}$. As our observations probe the entire annulus of WASP-121b during transit, any signals we retrieve are the result of an average over the limb. If, for example, clouds were blocking the side of the limb rotating towards us, this could result in an overall redshift being detected, though we note that it would seem very odd for this to happen to only some of the many species we have found evidence for. Significant cloud mass is also not expected in UHJs due to the high temperatures preventing condensates from forming, and this would be especially true for the hotter, blueshifted evening terminator. Nevertheless, GCMs by Flowers et al. (2019) for the cooler hot Jupiter HD 189733b show that a combination of winds, rotation, and clouds can lead to velocity shifts of $\pm 10 \mathrm{~km} \mathrm{~s}^{-1}$ in different regions of the limb, so this explanation lies within the bounds of theoretical possibility. Also, Ehrenreich et al. (2020) resolve their detection of Fe I in the atmosphere of the UHJ WASP-76b to have a blueshift of $-11 \mathrm{~km} \mathrm{~s}^{-1}$ on the 'evening' terminator and detect no signal from the nightside close to the morning terminator, which they attribute to the condensation of iron across the cooler nightside. A chemical gradient across the surface of the nightside of hot Jupiters is predicted by theory (e.g. Komacek \& Showman 2016; Bell \& 
Cowan 2018) and could potentially explain both the large blue- and redshifts seen in our data, depending on the exact chemistry involved. It is possible that using spectral models based on 3D atmospheric circulation models could result in stronger detections, as was found for emission in infrared by Beltz et al. (2021), and help uncover the cause of these offsets.

However, we find it more likely that the source of this variance in $v_{\text {sys }}$ is simply due to the inherent wavelength instability of UVES. During the alignment process outlined in Section 2, we see orderto-order variation in the wavelength solution for the blue arm of $\sim 3 \mathrm{~km} \mathrm{~s}^{-1}$. While we attempt to correct this with alignment to a stellar spectrum, it is possible that some variation remains, and we additionally make no attempt to refine the dispersion in each order. Also, the red arm was not corrected for order-to-order variation due to both its smaller amplitude and the difficulty of aligning telluric-free orders to the telluric spectrum used for alignment. If, for example, some orders are more divergent from the correct wavelength solution than others, then species with the majority of their lines in these orders would naturally present signals with variant $v_{\text {sys }}$ offsets. This more prosaic explanation is supported by the fact that our strongest signals are all detected at a $v_{\text {sys }}$ offset broadly consistent with previous works.

\section{CONCLUSIONS}

We have presented the results of a broad search for atomic species in the atmosphere of the UHJ WASP-121b using high-resolution spectroscopy. Using standard cross-correlation methodology on a single transit observation taken with UVES, we recovered potential signals for 17 neutral and ionized atomic species. Using five detection criteria, we confirm strong detections of Fe I, Ca II, CrI, VI, and $\mathrm{Ca}$, and tentative detections of $\mathrm{HI}, \mathrm{KI}$, and Sc II. We also uncover weak evidence for Mn I, Co II, Ni I, and Co I, and very weak hints of $\mathrm{Cu}$ I, Sr I, V II, Ti I, and Sr II. Technically insignificant yet potentially interesting signals of Fe II, Mg I, and Ti II are also discussed.

We have therefore presented independent confirmation or further evidence for previous detections of CrI, VI, CaI, and Ni I made by Ben-Yami et al. (2020) and Hoeijmakers et al. (2020b) using HARPS, and for the detections of $\mathrm{KI}$ and exospheric Ca II made by Borsa et al. (2021) using ESPRESSO. We additionally presented evidence of exospheric HI, previously found by Cabot et al. (2020) using HARPS, and present a novel detection of Sc II at $4.2 \sigma$. Finally, we have shown via injection tests that our methodology is sensitive to Y I, Y II, RbI, ScI, and CrII, species for which we do not find significant evidence, though we decline to set detection limits upon these species due the degeneracies present. The detection of such a wide range of atomic species allows us to begin to set constraints on the temperature and refractory properties of WASP-121b, and provides a useful starting point for more in-depth characterization of the exoplanet atmosphere.

The success of our search, in both confirming previous detections and in recovering a large number of potential signals, echoes that of previous successful broad species searches made in high-resolution for UHJs such as KELT-9b (Hoeijmakers et al. 2018, 2019) and KELT-20b/MASCARA-2b (Casasayas-Barris et al. 2019; Nugroho et al. 2020a). Our recovery of a variety of signals in a single transit is highly encouraging, and implies that the potential of highresolution spectroscopy for detecting atomic species in UHJs is as yet mostly untapped. However, although we have presented a large number of potential signals in this work, we emphasize that many of them are extremely weak or tentative. Future work using a greater number of transits, or with instruments with higher resolution and stability such as VLT/ESPRESSO, is encouraged to investigate the potential presence of many of the species presented herein, as well as to further investigate the velocity offsets of each detected species (e.g. Ehrenreich et al. 2020). We have also refrained from placing any solid constraints on the abundances of our detected species, or on the atmospheric structure of WASP-121b. Our removal of the continuum prevents the measurement of any pressure-sensitive features that would allow the degeneracies between abundances, temperature, scattering properties, and reference radius/pressure to be broken, and the cross-correlation method is not sensitive to changes in amplitude caused by differing scale heights. New and more complex approaches to high-resolution spectroscopic analysis have emerged in recent years, including the combination of lowand high-resolution spectroscopy (Brogi et al. 2017; Pino et al. 2018), principled statistical frameworks and likelihood mapping (Brogi \& Line 2019; Gibson et al. 2020; Hood et al. 2020; Nugroho et al. 2020b), machine learning (Fisher et al. 2020), and Doppler tomography (Watson et al. 2019; Matthews et al., in preparation). These sophisticated methods enable more stringent constraints to be placed on atmospheric parameters, and broad species searches such as ours present a starting point for future work using these sophisticated and more computationally intensive methods to further categorize the atmosphere of WASP-121b.

\section{ACKNOWLEDGEMENTS}

The authors are very grateful to the anonymous referee for suggestions and observations. We would like to thank Laura McKemmish for many useful comments. This work is based on observations collected at the European Organisation for Astronomical Research in the Southern Hemisphere under ESO programme 098.C-0547. NPG gratefully acknowledges support from Science Foundation Ireland and the Royal Society in the form of a University Research Fellowship. SKN and CAW would like to acknowledge support from UK Science and Technology Facilities Council grant ST/P000312/1. We are exceptionally grateful to the developers of the NUMPY, SCIPY, MATPLOTLIB, IPYTHON, SCIKIT-LEARN, ASTROPY, and NUMBA packages, which were used extensively in this work (Hunter 2007; Perez \& Granger 2007; Pedregosa et al. 2011; Astropy Collaboration et al. 2013; Lam, Pitrou \& Seibert 2015; Harris et al. 2020; Virtanen et al. 2020). The perceptually uniform scientific colour maps used in Figs 1 and 3-6 are by Fabio Crameri (Crameri, Shephard \& Heron 2020) and can be found at https://www.fabiocrameri.ch/colourmaps/. This work has made use of data from the European Space Agency (ESA) mission Gaia (https://www.cosmos.esa.int/gaia), processed by the Gaia Data Processing and Analysis Consortium (DPAC, https: //www.cosmos.esa.int/web/gaia/dpac/consortium). Funding for the DPAC has been provided by national institutions, in particular the institutions participating in the Gaia Multilateral Agreement.

\section{DATA AVAILABILITY}

The observations underlying this analysis are publicly available in the ESO Science Archive Facility under program name 098.C-0547. Other data will be shared on reasonable request to the corresponding author.

\section{REFERENCES}

Arcangeli J. et al., 2018, ApJ, 855, L30

Astropy Collaboration et al., 2013, A\&A, 558, A33

Astudillo-Defru N., Rojo P., 2013, A\&A, 557, A56 
Basri G., Mohanty S., Allard F., Hauschildt P. H., Delfosse X., Martín E. L., Forveille T., Goldman B., 2000, ApJ, 538, 363

Beatty T. G., Madhusudhan N., Tsiaras A., Zhao M., Gilliland R. L., Knutson H. A., Shporer A., Wright J. T., 2017, AJ, 154, 158

Bell T. J., Cowan N. B., 2018, ApJ, 857, L20

Beltz H., Rauscher E., Brogi M., Kempton E. M. R., 2021, AJ, 161, 1

Ben-Jaffel L., 2007, ApJ, 671, L61

Ben-Yami M., Madhusudhan N., Cabot S. H. C., Constantinou S., Piette A., Gandhi S., Welbanks L., 2020, ApJ, 897, L5

Benneke B., Seager S., 2012, ApJ, 753, 100

Birkby J. L., de Kok R. J., Brogi M., de Mooij E. J. W., Schwarz H., Albrecht S., Snellen I. A. G., 2013, MNRAS, 436, L35

Birkby J. L., de Kok R. J., Brogi M., Schwarz H., Snellen I. A. G., 2017, AJ, 153,138

Borsa F. et al., 2021, A\&A, 645, A24

Bourrier V. et al., 2020a, A\&A, 635, A205

Bourrier V. et al., 2020b, A\&A, 637, A36

Brogi M., Giacobbe P., Guilluy G., de Kok R. J., Sozzetti A., Mancini L., Bonomo A. S., 2018, A\&A, 615, A16

Brogi M., Line M. R., 2019, AJ, 157, 114

Brogi M., Line M., Bean J., Désert J. M., Schwarz H., 2017, ApJ, 839, L2

Brogi M., Snellen I. A. G., de Kok R. J., Albrecht S., Birkby J., de Mooij E. J. W., 2012, Nature, 486, 502

Cabot S. H. C., Madhusudhan N., Hawker G. A., Gandhi S., 2019, MNRAS, 482,4422

Cabot S. H. C., Madhusudhan N., Welbanks L., Piette A., Gandhi S., 2020, MNRAS, 494, 363

Casasayas-Barris N. et al., 2018, A\&A, 616, A151

Casasayas-Barris N. et al., 2019, A\&A, 628, A9

Colón K. D., Ford E. B., Redfield S., Fortney J. J., Shabram M., Deeg H. J., Mahadevan S., 2012, MNRAS, 419, 2233

Crameri F., Shephard G. E., Heron P. J., 2020, Nat Commun., 11, 5444

Daylan T. et al., 2021, AJ, 161, 131

Dekker H., D’Odorico S., Kaufer A., Delabre B., Kotzlowski H., 2000, in Iye M., Moorwood A. F., eds, Proc. SPIE Vol. 4008, Optical and IR Telescope Instrumentation and Detectors. SPIE, Bellingham, p. 534

Delrez L. et al., 2016, MNRAS, 458, 4025

Ehrenreich D. et al., 2020, Nature, 580, 597

Espinoza N. et al., 2019, MNRAS, 482, 2065

Esteves L. J., de Mooij E. J. W., Jayawardhana R., Watson C., de Kok R., 2017, AJ, 153, 268

Evans T. M. et al., 2016, ApJ, 822, L4

Evans T. M. et al., 2017, Nature, 548, 58

Evans T. M. et al., 2018, AJ, 156, 283

Fisher C., Hoeijmakers H. J., Kitzmann D., Márquez-Neila P., Grimm S. L., Sznitman R., Heng K., 2020, AJ, 159, 192

Flowers E., Brogi M., Rauscher E., Kempton E. M. R., Chiavassa A., 2019, AJ, 157, 209

Fortney J. J., Lodders K., Marley M. S., Freedman R. S., 2008, ApJ, 678, 1419

Fortney J. J., Shabram M., Showman A. P., Lian Y., Freedman R. S., Marley M. S., Lewis N. K., 2010, ApJ, 709, 1396

Fossati L. et al., 2010, ApJ, 714, L222

Fu G. et al., 2020, AJ, preprint (arXiv:2005.02568)

Gaia Collaboration, 2016, A\&A, 595, A1

Gaia Collaboration, 2018, A\&A, 616, A1

Gibson N. P. et al., 2020, MNRAS, 493, 2215

Gibson N. P., Aigrain S., Barstow J. K., Evans T. M., Fletcher L. N., Irwin P. G. J., 2013a, MNRAS, 428, 3680

Gibson N. P., Aigrain S., Barstow J. K., Evans T. M., Fletcher L. N., Irwin P. G. J., 2013b, MNRAS, 436, 2974

Gibson N. P., de Mooij E. J. W., Evans T. M., Merritt S., Nikolov N., Sing D. K., Watson C., 2019, MNRAS, 482, 606

Grimm S. L., Heng K., 2015, ApJ, 808, 182

Harris C. R. et al., 2020, Nature, 585, 357

Haswell C. A. et al., 2012, ApJ, 760, 79

Helling C., Gourbin P., Woitke P., Parmentier V., 2019, A\&A, 626, A133

Heng K., Kitzmann D., 2017, MNRAS, 470, 2972
Herman M. K., de Mooij E. J. W., Jayawardhana R., Brogi M., 2020, AJ, 160, 93

Hoeijmakers H. J. et al., 2018, Nature, 560, 453

Hoeijmakers H. J. et al., 2019, A\&A, 627, A165

Hoeijmakers H. J. et al., 2020a, A\&A, 641, A120

Hoeijmakers H. J. et al., 2020b, A\&A, 641, A123

Høg E. et al., 2000, A\&A, 355, L27

Hood C. E. et al., 2020, AJ, 160, 198

Hubeny I., Burrows A., Sudarsky D., 2003, ApJ, 594, 1011

Hunter J. D., 2007, Comput. Sci. Eng., 9, 90

Husser T. O., Wende-von Berg S., Dreizler S., Homeier D., Reiners A., Barman T., Hauschildt P. H., 2013, A\&A, 553, A6

Jones A., Noll S., Kausch W., Szyszka C., Kimeswenger S., 2013, A\&A, 560, A91

Kataria T., Sing D. K., Lewis N. K., Visscher C., Showman A. P., Fortney J. J., Marley M. S., 2016, ApJ, 821, 9

Kesseli A. Y., Snellen I. A. G., Alonso-Floriano F. J., Molliere P., Serindag D. B., 2020, AJ, 160, 228

Khalafinejad S. et al., 2017, A\&A, 598, A131

Kirk J., Wheatley P. J., Louden T., Doyle A. P., Skillen I., McCormac J., Irwin P. G. J., Karjalainen R., 2017, MNRAS, 468, 3907

Kitzmann D. et al., 2018, ApJ, 863, 183

Komacek T. D., Showman A. P., 2016, ApJ, 821, 16

Kurucz R. L., 2018, in Mendoza C., Turck-Chiéze S., Colgan J., eds, ASP Conf. Ser. Vol. 515, Workshop on Astrophysical Opacities. Astron. Soc. Pac., San Francisco, p. 47

Lam S. K., Pitrou A., Seibert S., 2015, in LLVM '15: Proceedings of the Second Workshop on the LLVM Compiler Infrastructure in HPC. Association for Computing Machinery (ACM), New York, p. 1

Lecavelier Des Etangs A., Pont F., Vidal-Madjar A., Sing D., 2008, A\&A, 481, L83

Lockwood A. C., Johnson J. A., Bender C. F., Carr J. S., Barman T., Richert A. J. W., Blake G. A., 2014, ApJ, 783, L29

Lodders K., 2002, ApJ, 577, 974

Lothringer J. D., Barman T., 2019, ApJ, 876, 69

Lothringer J. D., Barman T., Koskinen T., 2018, ApJ, 866, 27

Lothringer J. D., Fu G., Sing D. K., Barman T. S., 2020, ApJ, 898, L14

Mallonn M., Köhler J., Alexoudi X., von Essen C., Granzer T., Poppenhaeger K., Strassmeier K. G., 2019, A\&A, 624, A62

Mandel K., Agol E., 2002, ApJ, 580, L171

May E. M., Zhao M., Haidar M., Rauscher E., Monnier J. D., 2018, AJ, 156, 122

Merritt S. R. et al., 2020, A\&A, 636, A117

Mikal-Evans T. et al., 2019, MNRAS, 488, 2222

Mikal-Evans T., Sing D. K., Kataria T., Wakeford H. R., Mayne N. J., Lewis N. K., Barstow J. K., Spake J. J., 2020, MNRAS, 496, 1638

Noll S., Kausch W., Barden M., Jones A. M., Szyszka C., Kimeswenger S., Vinther J., 2012, A\&A, 543, A92

Nugroho S. K., Gibson N. P., de Mooij E. J. W., Herman M. K., Watson C. A., Kawahara H., Merritt S. R., 2020b, ApJ, 898, L31

Nugroho S. K., Gibson N. P., de Mooij E. J. W., Watson C. A., Kawahara H., Merritt S., 2020a, MNRAS, 496, 504

Nugroho S. K., Kawahara H., Masuda K., Hirano T., Kotani T., Tajitsu A., 2017, AJ, 154, 221

Parmentier V. et al., 2018, A\&A, 617, A110

Parmentier V., Fortney J. J., Showman A. P., Morley C., Marley M. S., 2016, ApJ, 828, 22

Parmentier V., Showman A. P., Lian Y., 2013, A\&A, 558, A91

Pedregosa F. et al., 2011, J. Machine Learning Res., 12, 2825

Perez F., Granger B. E., 2007, Comput. Sci. Eng., 9, 21

Pino L. et al., 2018, A\&A, 612, A53

Salz M., Schneider P. C., Fossati L., Czesla S., France K., Schmitt J. H. M. M., 2019, A\&A, 623, A57

Sedaghati E. et al., 2016, A\&A, 596, A47

Seidel J. V. et al., 2020, A\&A, 641, L7

Sing D. K. et al., 2011, A\&A, 527, A73

Sing D. K. et al., 2013, MNRAS, 436, 2956

Sing D. K. et al., 2019, AJ, 158, 91 
Snellen I. A. G., 2004, MNRAS, 353, L1

Snellen I. A. G., de Kok R. J., de Mooij E. J. W., Albrecht S., 2010, Nature, 465, 1049

Spiegel D. S., Silverio K., Burrows A., 2009, ApJ, 699, 1487

Stangret M., Casasayas-Barris N., Pallé E., Yan F., Sánchez-López A., LópezPuertas M., 2020, A\&A, 638, A26

Tamuz O., Mazeh T., Zucker S., 2005, MNRAS, 356, 1466

Tsiaras A. et al., 2018, AJ, 155, 156

Turner J. D. et al., 2020, ApJ, 888, L13

Vidal-Madjar A. et al., 2013, A\&A, 560, A54

Vidal-Madjar A., Lecavelier des Etangs A., Désert J. M., Ballester G. E., Ferlet R., Hébrard G., Mayor M., 2003, Nature, 422, 143
Virtanen P. et al., 2020, Nat. Methods, 17, 261

Wakeford H. R. et al., 2017, ApJ, 835, L12

Watson C. A., de Mooij E. J. W., Steeghs D., Marsh T. R., Brogi M., Gibson N. P., Matthews S., 2019, MNRAS, 490, 1991

Wilson J. et al., 2020, MNRAS, 497, 5155

Wilson J., Gibson N. P., Lothringer J. D., Sing D. K., Mikal-Evans T., de Mooij E. J. W., Nikolov N., Watson C. A., 2021, MNRAS, 503, 4787

Yan F. et al., 2019, A\&A, 632, A69

This paper has been typeset from a $\mathrm{T}_{\mathrm{E}} \mathrm{X} / \mathrm{L} \mathrm{T} \mathrm{E} \mathrm{X}$ file prepared by the author. 